\title{
SELFSIMILAR BLOWUP OF UNSTABLE THIN-FILM EQUATIONS
}

\author{
D. SLEPČEV AND M.C. PUGH
}

\begin{abstract}
We study selfsimilar blowup of long-wave unstable thin-film equations with critical powers of nonlinearities: $u_{t}=-\left(u^{n} u_{x x x}+u^{n+2} u_{x}\right)_{x}$. We show that the equation cannot have selfsimilar solutions (with zero contact angles) that blow up in finite time if $n \geq 3 / 2$. We show that for $0<n<3 / 2$ there are compactly supported, symmetric, selfsimilar solutions (with zero contact angles) that blow up in finite time. Moreover there exist families of these solutions with any number of local maxima. We also study the asymptotic behaviour of these selfsimilar solutions as $n$ approaches $3 / 2$ and obtain a sharp lower bound on the height of solutions one time unit before the blowup. We also prove qualitative properties of solutions; for example, the profile of a selfsimilar solution that blows up in finite time can always be bounded from below by a compactly supported steady state.
\end{abstract}

\section{INTRODUCTION}

Long-wave unstable thin-film equations model the dynamics of a thin film of viscous fluid that is being acted upon by two competing forces. For example, if the film is hanging from a flat surface then gravity tends to make the film increase the area of its air/liquid interface, as pendant drops begin to form, while surface tension tends to make the film decrease the area of its air/liquid interface. As both forces act simultaneously, their competing effects lead to a greater variety of short-time and long-time behaviors than would occur if only one force were present. The thin-film dynamics can be modeled by:

$$
u_{t}=-\nabla \cdot(f(u) \nabla \Delta u)-\nabla \cdot(g(u) \nabla u) .
$$

Here, the air/liquid interface is represented as the graph of a function: $z=u(x, y, t)$ where $u \geq 0$ at all points in the domain. The coefficient $f(u)$ models the effect of surface tension. It is nonnegative with a degeneracy at zero: $f(0)=0$ and $f(z)>0$ if $z>0$. The coefficient $g(u)$ models additional forces, such as gravity, and is also nonnegative. In the case of a thin film hanging from a flat surface in the presence of gravity, $f(z)=z^{3}$ and $g(z)=z^{3}$ [13]. We refer the reader to survey articles for more information on the modeling and the physics of thin liquid films [19,20].

In [8], Bertozzi and Pugh consider the case where the film thickness is uniform in one direction: $z=u(x, t)$. They make a conjecture as to the balance between the fourthorder stabilizing term and the second-order destabilizing term, identifying subcritical,

Date: November 22, 2004. 
critical, and supercritical regimes. For power-law coefficients, the general equation (1) becomes

$$
u_{t}=-\left(u^{n} u_{x x x}\right)_{x}-\left(u^{m} u_{x}\right)_{x} .
$$

The subcritical regime corresponds to $m<n+2$, the critical regime to $m=n+2$, and the supercritical regime to $m>n+2$. They conjectured that in the subcritical regime nonnegative solutions will have uniformly bounded $L^{\infty}$-norms and that in the supercritical regime there exist initial data that yield solutions whose $L^{\infty}$ norm blow up in finite time. In [8], they proved the subcritical part of the conjecture. Numerical simulations of the initial value problem confirm the conjecture in the supercritical case, and suggest that when blowup occurs, the solution focuses in a selfsimilar manner at isolated points in space. In [9], they considered the $n=1$ case and proved that in the critical and supercritical regimes there exist initial data that yield solutions whose $L^{\infty}$-norm blows up in finite time.

The existence theory in $[8,9]$ hinges on the energy that when $m \neq n-1$ and $m \neq n-2$ has the form:

$$
\mathcal{E}(t)=\int \frac{1}{2} u_{x}^{2}(x, t)-\frac{1}{(m-n+2)(m-n+1)} u^{m-n+2}(x, t) d x,
$$

which is dissipated by the evolution: $\mathcal{E}(t) \leq \mathcal{E}(0)$ for $t>0$. In the subcritical regime, one can use a Gagliardo-Nirenberg inequality to prove that given initial data $u_{0}$, there exists a constant $C$ determined by the mass of $u_{0}$ such that $\|u(\cdot, t)\|_{H^{1}} \leq 4 \mathcal{E}(t)+C$ at almost all times. Hence the dissipation of $\mathcal{E}$ ensures that the $H^{1}$-norm is bounded and blowup cannot happen. In the critical regime,

$$
u_{t}=-\left(u^{n} u_{x x x}\right)_{x}-\left(u^{n+2} u_{x}\right)_{x}
$$

Witelski, Bernoff, and Bertozzi [25] found that a sharp Sz.-Nagy inequality [23, 18] ensures that initial data with sufficiently small initial mass cannot blow up. Specifically,

$$
\int u^{4}(x, t) d x \leq \frac{9}{4 \pi^{2}}\left(\int|u(x, t)| d x\right)^{2} \int u_{x}^{2}(x, t) d x .
$$

For nonnegative solutions that conserve their mass, this implies

$$
\left(\frac{1}{2}-\frac{3\left(\int u_{0}\right)^{2}}{16 \pi^{2}}\right) \int u_{x}^{2}(x, t) d x \leq \mathcal{E}(t) \leq \mathcal{E}(0) .
$$

If the initial data has sufficiently small mass, $\int u_{0} d x<2 \pi \sqrt{2 / 3}=: M_{c}$, the lefthand side is positive, allowing the energy $\mathcal{E}$ to be used in an a priori bound of the $H^{1}$-norm of $u$. The existence theory [8] then follows with weak solutions existing for all time and having zero contact angles at almost all times (c.f. [25, §2] for another argument for the criticality of $M_{c}$ in the $n=1$ case).

For the interested reader, we provide references to recent articles that bear on the existence theory and provide additional references to other work. First, for equation (1) with $f(u)=u$ and $g(u)=0$, variational methods yield solutions that are uniform in one direction $(u(x, y, t)=u(x, t))$ and can have fixed nonzero contact angles [21]. For equation (1) with $g(u)=0$, if one seeks solutions that are uniform in one direction 
there are existence and finite speed of propagation results based on energy methods (see $[3,16]$ and references therein). If one considers solutions of equation (1) with $g(u) \leq 0$ in higher dimensions $(u(x, y, t)$ and $u(x, y, z, t))$, there are existence results, finite speed of propagation results, and waiting time results (see $[12,15]$ and references therein).

1.1. Special Solutions in the Critical Regime. In this article, we refer to contact lines and contact angles and so we first define these terms. If $v \in C(\mathbb{R})$ and $v \geq 0$ then we say that $x_{0}$ is on the contact line if $v\left(x_{0}\right)=0$ and $v>0$ on $\left(x_{0}-\varepsilon, x_{0}\right)$ or $\left(x_{0}, x_{0}+\varepsilon\right)$ for some $\varepsilon>0$. (Note that $v$ could be positive to both sides of $x_{0}$.) If $v>0$ on a left neighborhood of $x_{0}$ and $\lim _{x \rightarrow x_{0}-} v^{\prime}(x)$ exists then the contact angle to the left of $x_{0}$ is the arctangent of this limit. Similarly, if $v>0$ on a right neighborhood of $x_{0}$ and

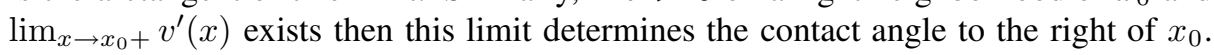
(Note that the contact angles to the left of $x_{0}$ and to the right of $x_{0}$ might not be equal.) If $v>0$ holds on only one side of $x_{0}$ then we will refer to the contact angle at $x_{0}$ and will not specify whether it is to the right or left of $x_{0}$.

One line of inquiry [17] involves a study of the steady states of equation (2). Three types of steady states have been found: constant steady states, positive periodic steady states, and "droplet" steady states. Droplet steady states correspond to $u \geq 0$ where $u$ is supported on an interval $[a, b]$, is increasing on $[a, c]$, is decreasing on $[c, b]$, and has equal contact angles: $u_{x}(a)=-u_{x}(b)$. There is a natural scaling which allows steady states to be rescaled to create other steady states. In the critical regime, $m=n+2$, the mass of the zero-contact-angle droplet steady state is $M_{c}=2 \pi \sqrt{2 / 3}$ and the rescaling preserves the mass $[17, \S 3.1 .1]$. This is relevant because the existence theory in $[8,9]$ for equation (2) yields solutions that have zero contact angles at almost all times. And so these zero-contact-angle steady states are admissible weak solutions.

Another line of research is the study of selfsimilar solutions of equation (3). The scaling invariance of the equation $\left(x \rightarrow \lambda x, u \rightarrow u / \lambda, t \rightarrow \lambda^{n+4} t\right)$, suggest the existence of solutions of the form:

$$
u(x, t)=(T+\sigma t)^{-1 /(n+4)} U\left(\frac{x}{(T+\sigma t)^{1 /(n+4)}}\right)
$$

where $\sigma=1$ corresponds to a source-type solution and $\sigma=-1$ corresponds to a solution which blows up at time $t=T$. The profile $U$ of the selfsimilar solution satisfies the ODE

$$
-\frac{\sigma}{n+4}(z U(z))^{\prime}=-\left(U^{n}(z) U^{\prime \prime \prime}(z)+U^{n+2}(z) U^{\prime}(z)\right)^{\prime}
$$

where $z:=x /(T+\sigma t)^{1 /(n+4)}$. If $U$ is symmetric about $z=0$ or if there is a point $z_{0}$ on the contact line such that the flux $U^{n} U^{\prime \prime \prime}+U^{n+2} U^{\prime}$ goes to zero as $z \rightarrow z_{0}$, then one can integrate up to find

$$
-\frac{\sigma}{n+4} z U(z)=-U^{n}(z) U^{\prime \prime \prime}(z)-U^{n+2}(z) U^{\prime}(z) .
$$

In the interior of its support, the profile $U$ satisfies the ordinary differential equation

$$
U^{\prime \prime \prime}(z)=\frac{\sigma}{n+4} z U^{1-n}(z)-U^{2}(z) U^{\prime}(z) \quad \text { wherever } U>0 .
$$


In [1], Beretta used a shooting method analogous to that in [6] to prove the existence of selfsimilar source-type $(\sigma=1)$ solutions that have compact support and zero contact angles. In this case, there are monotonicity properties that make the analysis tractable. Specifically, if $z_{0} \geq 0$ is a local maximum then equation (5) implies $U^{\prime \prime \prime}(z)>0$ if $U^{\prime}(z)<0$ and $z>z_{0}$. Our methods in $\S 4$ show that any such solution must have mass less than $M_{c}$.

Witelski, Bernoff, and Bertozzi [25] use asymptotics and numerics to study the $n=1$ case of equation (3). They find that all source-type selfsimilar solutions with compact support and zero contact angles have mass less than $M_{c}$ and that they are linearly stable to mass-preserving perturbations. Using the length of the support as a bifurcation parameter, they find that there is an upper bound on the possible length of the support of the droplet profile and that there are two regimes as the length tends to zero; in one regime the mass tends to zero and in the other regime, the mass tends to $M_{c}$. They also consider solutions that blow up in a selfsimilar manner in finite time. They find infinitely many profiles that are symmetric about $x=0$, compactly supported, and have zero contact angles. All these profiles have mass greater than $M_{c}$ and are distinguished by their number of local maxima. They find that the profiles with more than one local maximum are linearly unstable to mass-preserving perturbations and that the "droplet" profile is linearly stable modulo perturbations that would change the blowup time or the blowup point. Using the length of the support as a bifurcation parameter, they find that there is an upper bound on the possible length of the droplet profile and that as the length tends to zero the mass decreases to $M_{c}$ and as the length increases to the maximum possible length, the mass increases to an upper bound $M_{u}$.

1.2. Results. The analytical study of selfsimilar blow-up solutions is especially challenging because when $\sigma=-1$ equation (5) loses the convexity properties that are used in the analysis of the source-type solutions. For this reason, much of our analysis relies on the fact that the selfsimilar profiles $U$ are "very close" to the steady-state solutions and then taking advantage of fine information about these steady states. In the $n>1$ case, this becomes especially delicate near the contact line since the first term on the right-hand side of equation (5) diverges as $U \downarrow 0$ while the equation satisfied by the steady state ( $\sigma=0$ in equation (5)) has no such divergence.

Some of our results include

- If $n \geq 3 / 2$, there are no selfsimilar zero-contact-angle solutions that blow up in finite time (Theorem 4$)$.

- If $0<n<3 / 2$ there is a threshold $H_{n}$, that diverges as $n \uparrow 3 / 2$, such that there is no zero-contact-angle selfsimilar solution $u$ with $\max (u(x, 0)) \leq H_{n}$ that blows up at time $T=1$ (Theorem 9).

- If $0<n<3 / 2$ there is a threshold $\widetilde{H}_{n}$, that diverges as $n \uparrow 3 / 2$, such that if $H \geq \widetilde{H}_{n}$ then there is a selfsimilar solution $u$ that has compact support and zero contact angles, has $\max (u(x, 0))=H$, and blows up at time $T=1$. (Theorems 11 and 19).

- If $0<n<3 / 2$ then given $\varepsilon>0$ there exists $H_{\varepsilon}$ such that if $H>H_{\varepsilon}$ then there is a selfsimilar solution $u$ with compact support and zero contact angles and 
$u(x, 0)$ is $\varepsilon$-close to a zero-contact-angle droplet steady state as measured in the $C^{2}$-norm (Corollaries 14 and 22).

- If $0<n<3 / 2$ then for each $k \in \mathbb{N}$ there exists a selfsimilar solution that is symmetric about $x=0$, has $k$ local maxima, compact support, zero contact angles and blows up in finite time (Theorems 29 and 30).

1.3. Related Work. Bernis, Peletier and Williams [6] consider source-type solutions of $u_{t}=-\left(u^{n} u_{x x x}\right)_{x}$. They study the ODE $U^{\prime \prime \prime}=x U^{1-n}$ that the profile $U$ must satisfy. The ODE is tractable in that it has helpful convexity properties $\left(U^{\prime \prime \prime}(x)>0\right.$ for $x>0$ and $U^{\prime \prime \prime}(x)<0$ for $x<0$ ) although it does have the difficulty that it is singular at $U=0$ if $n>1$. Using shooting methods, they prove that if $0<n<3$ there exist source-type solutions with compact support and zero contact angles. These solutions are symmetric, unique, and cannot exist if $n \geq 3$.

Ferreira and Bernis [14] study the equation in higher space dimensions: $u_{t}=$ $-\nabla \cdot\left(u^{n} \nabla \Delta u\right)$. They consider radially-symmetric solutions and find that if $0<n<3$ there exist compactly supported source-type solutions with zero contact angles. The analytical difficulty is that in dimensions greater than 1, the Laplacian in radial coordinates has a singularity at $r=0$. Their approach is to rescale the problem to a fixed interval, regularize the boundary data, solve the regularized problem via a fixed point theorem, and then take a limit of the regularized solutions to find the desired solution. In addition, they prove the uniqueness of the solution in an elegant way, without using the fixed point argument. An obstacle to applying their approach to the evolution equation (3) arises from the rescaling. They first rescale the problem so that a radial solution with compact support $[0, R]$ becomes a solution supported on $[0,1]$, where the rescaled solution satisfies a specific boundary value problem. Equation (3) has fewer invariances than their equation, and their rescaling approach leaves an unknown parameter in the rescaled equation. In [4], they carefully analyze the $n \uparrow 3$ limit. Letting $u_{n}(r, t)$ denote the radially symmetric source-type selfsimilar solution supported on $\left[0, a_{n}\right]$, they find that the rescaled object $a_{n}^{D} u_{n}\left(a_{n} x, t\right)$ converges to a parabolic profile with nonzero contact angle as $n \uparrow 3$ (here $D$ is the spatial dimension).

Bernis, Hulshof and King [5] consider $u_{t}=-\left(u^{n} u_{x x x}\right)_{x}$ on the half-line $[0, \infty)$. At $x=0$ they consider two types of boundary conditions: no-flux and zero contact angle. For the no-flux boundary conditions, $u(0)=u^{n}(0) u_{x x x}(0)=0$, selfsimilar source-type solutions are shown to exist for $0<n<3$. These solutions are compactly supported on $[0, a]$ with zero contact angle at $x=a$. Such solutions cannot exist if $n \geq 3$ [6]. For the zero contact angle boundary conditions, $u(0)=u_{x}(0)=0$, there is no mass-conservation (the liquid "spills" over the edge at $x=0$ ). In this case, selfsimilar solutions ("dipole solutions") satisfy a selfsimilar problem of "second kind": a nonlinear eigenvalue problem in which one of the scaling exponents, $\alpha$, is determined simultaneously with the solution $u$. In the $n=1$ case, the first moment of the solution is a conserved quantity, allowing them to determine the value of $\alpha$ and then prove the existence of dipole solutions. They also prove that the dipole problem cannot have solutions if $n \geq 2$. The authors present three methods for solving the ODEs that the profiles $U$ must satisfy. They use fixed point arguments (as in [14]) to prove 
the existence of the source-type solutions. They use a shooting argument to find the dipole solutions in the $n=1$ case. They then give different proof using a dynamical system approach which allows them to prove the uniqueness of the dipole solutions. In this approach, they transform the problem into a three-dimensional dynamical system in which the profile $U$ corresponds to a heteroclinic orbit. The dynamical system is nontrivial, with one of the critical points being at infinity, and its analysis is involved.

In [10], Bowen, Hulshof and King continue their work on dipole solutions, studying the $0<n<2$ range in detail. They use the dynamical systems approach to study the problem; since $n \neq 1$ a four-dimensional dynamical system is needed. To show that connecting orbits exist they use a numerical construction based on a "shooting method program". In particular, they study the $n \rightarrow 1$ and $n \uparrow 2$ limit in detail via asymptotics to study "almost conserved quantities" and their dependence on $n$. Specifically, if $n=1$ the first moment is conserved by the dipole solution and is almost conserved by the dipole solutions for $n$ close to 1 . Similarly, if $n=2$ there is a selfsimilar solution that conserves mass and mass is almost conserved by the dipole solutions for $n$ close to 2 . Their $n \uparrow 2$ study via asymptotics and computations yields results that are somewhat similar in spirit to Corollaries 14 and 22. In [24], van den Berg et al. consider dipole solutions on a fixed interval $[0,1]$. In this case, separable solutions are the exact solutions dictated by scaling arguments. The authors prove the existence of a separable solution if $n<2$. The solution is unique and is symmetric about $x=1 / 2$. The existence and uniqueness is proven using a shooting method combined with elegant comparison arguments involving "super-sub pairs". They also study the $n \uparrow 2$ limit and find that the solution, after an appropriately rescaling, converges to a parabolic profile with nonzero contact angles.

Singularity formation in long-wave unstable thin-film equations with power nonlinearities has been studied in the context of film rupture $(u \downarrow 0)$. In particular the equation with $n=3$ and $m=-1$ has been studied by Zhang and Lister [28], and Witelski and Bernoff $[26,27]$. It was shown both numerically and by asymptotic analysis that the film rupture has a selfsimilar structure. The selfsimilar profiles have been computed $[28,26]$ and the stability of the simplest profile has been shown [26, 27].

\section{STEAdy STATeS}

In Section 4, we will prove that at one time-unit before blowup, the profile of a compactly supported, symmetric, selfsimilar solution with zero contact angles is very close to that of a compactly supported steady state with zero contact angles, with the significant differences between the two occurring near the contact line. For this reason, we start by analyzing the steady states of the evolution equation (3).

The steady states are translation invariant. Three types of nonconstant steady states have been found and studied: positive periodic, compactly supported with zero contact angles, and compactly supported with equal nonzero contact angles [17]. The simplest compactly supported steady state is a "droplet" - supported on $[a, b]$, symmetric about $x=(a+b) / 2$, where it has a global maximum and decreasing to zero as $x$ increases to $b$ or decreases to $a$. In fact, if a steady state is supported on $[a, b]$, is positive on 
$(a, b)$, and has equal contact angles at $x=a, b$ then it must be a droplet steady state [17]. A collection of droplet steady states with supports $\left\{\left[a_{\alpha}, b_{\alpha}\right]\right\}, \alpha \in I$ is also a steady state if $\left(a_{\alpha}, b_{\alpha}\right) \cap\left(a_{\beta}, b_{\beta}\right)=\emptyset$ for $\alpha \neq \beta$. However such configurations will not be relevant in this article. If we refer to a steady state with compact support, we are implicitly assuming the steady state is a droplet. Further, in considering steady states with compact support we will implicitly assume they have zero contact angles since such regularity is consistent with the existence theory for equation (2) found in $[8,9]$.

If $V$ is a steady state, and $[a, b]$ is the support of $V$, then $V \in C^{\infty}([a, b])$. If one takes $x_{0} \in(a, b)$, one can view $V$ as the solution of the initial value problem

(6)

$$
V^{\prime \prime \prime}(x)=-V^{2}(x) V^{\prime}(x) ; \quad V\left(x_{0}\right)=H>0, \quad V^{\prime}\left(x_{0}\right)=\xi \leq 0, \quad V^{\prime \prime}\left(x_{0}\right)=\gamma<0
$$

(If $V$ is a nonconstant steady state, one can always choose $x_{0}$ such that $V^{\prime}\left(x_{0}\right) \leq 0$ and $V^{\prime \prime}\left(x_{0}\right)<0$.) Whenever we view the solution $V$ as arising from an initial value problem, we are implicitly assuming the solution has been defined on its maximal interval of positivity (which is precisely $(a, b)$ ). In other words we "stop" the solution once it reaches zero. Integrating equation (6) yields

$$
V^{\prime \prime}(x)=\gamma+\frac{H^{3}}{3}-\frac{V(x)^{3}}{3} ; \quad V\left(x_{0}\right)=H, \quad V^{\prime}\left(x_{0}\right)=\xi .
$$

Intuitively, if $\gamma$ is "too negative" then $V$ will have compact support and nonzero contact angles and if $\gamma$ is "not sufficiently negative" then $V$ will be positive and periodic. In fact, there is a unique value of $\gamma$ for which $V$ has compact support and zero contact angles.

Since $\xi \leq 0$ and $\gamma<0, V^{\prime}<0$ on $\left(x_{0}, x_{0}+\varepsilon\right)$ for some $\varepsilon>0$. Let $x_{\min }$ be the first $x$ greater than $x_{0}$ such that $V^{\prime}\left(x_{\min }\right)=0$ or $V\left(x_{\min }\right)=0$ :

$$
x_{\text {min }}:=\sup \left\{x \mid V^{\prime}<0 \text { and } V>0 \text { on }\left(x_{0}, x\right)\right\} \quad V_{\text {min }}:=V\left(x_{\text {min }}\right) .
$$

Then $V:\left[x_{0}, x_{\min }\right] \rightarrow\left[V_{\min }, H\right]$ is an invertible function; we denote its inverse by $x(V)$. The change of variables

$$
p(V):=\frac{d V}{d x}(x(V)) \quad \Longrightarrow \quad p(V) \frac{d p}{d V}(V)=\frac{d^{2} V}{d x^{2}}(x(V)) .
$$

transforms the second-order initial value problem (7) into the first-order initial value problem:

$$
p(V) \frac{d p}{d V}(V)=\gamma+\frac{H^{3}}{3}-\frac{V^{3}}{3} \quad \text { for } V \in\left(V_{\min }, H\right] ; \quad p(H)=\xi .
$$

We solve this exactly:

$$
p(V)=-\frac{1}{\sqrt{6}} \sqrt{6 \xi^{2}+V\left(H^{3}-V^{3}\right)-3\left(4 \gamma+H^{3}\right)(H-V)} .
$$

If $\xi=0$ and $V_{\min }>0$, this solution can be extended to a positive periodic solution on the entire real line. On the interval $\left[x_{0}, x_{\min }\right]+k x_{\min }, V$ is determined by

$$
p(V)=(-1)^{k} \frac{1}{\sqrt{6}} \sqrt{V\left(H^{3}-V^{3}\right)-3\left(4 \gamma+H^{3}\right)(H-V)} .
$$


If $\xi=0$ and $V_{\min }=0$, we reflect the solution about $x=x_{0}$ to find a compactly supported droplet solution on $\left[x_{0}-\left(x_{\min }-x_{0}\right), x_{\text {min }}\right]$.

If $\xi=0$ then for $\gamma=-H^{3} / 4, V_{\min }=0$ and $V^{\prime}\left(x_{\min }\right)=0$. This compactly supported solution with zero contact angles will play a special role; we denote it by $\bar{V}$ (with its dependence on $H$ being implicit). We further denote the corresponding $p$ and $x_{\min }$ by $\bar{p}$ and $\bar{x}_{\min }$. Note that if $x_{0}=0$

$$
x_{\text {min }}=-\int_{V_{\text {min }}}^{H} \frac{d x}{d V} d V=-\int_{V_{\text {min }}}^{H} \frac{1}{p(V)} d V .
$$

Therefore

$$
\bar{x}_{\text {min }}=\int_{0}^{H} \frac{\sqrt{6}}{\sqrt{V\left(H^{3}-V^{3}\right)}} d V=\frac{1}{H} \frac{2 \sqrt{2}}{\sqrt{3}} \frac{\Gamma\left(\frac{3}{2}\right) \Gamma\left(\frac{1}{6}\right)}{\Gamma\left(\frac{2}{3}\right)} \approx \frac{5.949}{H}
$$

and the mass $\left(L^{1}\right.$-norm) of the stationary solution, $\bar{m}$, is given by

$$
\frac{\bar{m}}{2}=\int_{0}^{H} x(\bar{V}) d \bar{V}=\int_{0}^{H} \frac{y}{-\bar{p}(y)} d y=\int_{0}^{H} \frac{\sqrt{6} y}{\sqrt{y\left(H^{3}-y^{3}\right)}} d y=\sqrt{\frac{2}{3}} \pi .
$$

Thus the compactly supported zero contact angle steady states satisfy the following scaling behavior: as their height increases, their width decreases, and their mass stays the same.

In Section 4, we show that for $H$ sufficiently large one can find $\gamma$ slightly larger than $-H^{3} / 4$ so that the solution of (5) with $\sigma=-1, U(0)=H, U^{\prime}(0)=0$, and $U^{\prime \prime}(0)=\gamma$ has compact support and zero contact angles. We do this by showing that if $\gamma \in\left[-H^{3} / 4,-H^{3} / 4+3 H\right]$ then $U$ will be close to $\bar{V}$. To do this we will need some simple estimates on solutions of (6). For all $H \geq 40$ and $\gamma \in\left[-\frac{H^{3}}{4},-\frac{H^{3}}{4}+3 H\right]$ the following hold:

(P1) $V_{\min } \in(0.9,1) \frac{12 \gamma+3 H^{3}}{H^{2}}$.

(P2) For all $V \in\left[V_{\min }, H\right],|p(V)|<\frac{1}{3} H^{2}$.

(P3) $x_{\text {min }} \leq \bar{x}_{\text {min }}<5.95 / H$ and if $\gamma<-H^{3} / 4+H$ then $x_{\text {min }} \in(5.8,5.95) / H$.

Property (P1) can be verified by evaluating $p(V)$ at the endpoints of the intervals, while showing property (P2) is a calculus exercise. To prove (P3) it is enough to note that $p(V)=-\frac{1}{\sqrt{6}} \sqrt{\left(V-V_{\text {min }}\right)(H-V)\left(H^{2}+H V+V^{2}+H V_{\min }+V_{\min } V+V_{\min }^{2}\right)}$ and use appropriate substitution and property (P1) to compare the integral for $x_{\min }$ with the integral for $\bar{x}_{\min }$.

\section{GENERAL PROPERTIES OF SELFSIMILAR SOLUTIONS}

We seek a selfsimilar solution that blows up at time $T=1$. In this case, $z=x$ at time $t=0$ and so we drop the $z$ notation from the introduction and seek solutions of the equation (5) with $\sigma=-1$ by studying the ODE initial value problem:

$$
U^{\prime \prime \prime}=-\frac{1}{n+4} x U^{1-n}-U^{2} U^{\prime} ; U\left(x_{0}\right)=H>0, U^{\prime}\left(x_{0}\right)=\xi, U^{\prime \prime}\left(x_{0}\right)=\gamma
$$


The integral form of equation (12) is:

$$
U^{\prime \prime}=\gamma+\frac{H^{3}}{3}-\frac{U^{3}}{3}-\frac{1}{n+4} \int_{x_{0}}^{x} s U(s)^{1-n} d s ; \quad U\left(x_{0}\right)=H, U^{\prime}\left(x_{0}\right)=\xi
$$

$U$ will be assumed to be defined by the ODE on the closure of its maximal interval of positivity: $a<x_{0}<b$. Note that when $n>1$ then $(a, b)$ is also the maximal interval on which the (classical) solution exists. After defining $U$ to be zero outside $[a, b]$, formula (4) yields a selfsimilar solution $u$ of the Cauchy problem for the thin-film equation (3). If $0 \in(a, b)$ then the solution focuses at $x=0$ as $t \rightarrow 1$. If $0 \notin(a, b)$ then the solution's support moves towards $x=0$ as $t \rightarrow 1$. (Note that if $U$ does not have zero contact angles then the resulting solution $u$ does not fit into the existence theory of $[8,9]$. Indeed, in this article we seek $U$ with zero contact angles.)

The above construction yields a selfsimilar solution with connected support. One could imagine constructing a selfsimilar solution with disconnected support as follows: seek $x_{0}$ and $\tilde{x}_{0}$ and initial data at $x_{0}$ and $\tilde{x}_{0}$ such that (12) has a solution with maximal interval of existence $(a, b) \ni x_{0}$ and $(\tilde{a}, \tilde{b}) \ni \tilde{x}_{0}$ and $[a, b] \cap[\tilde{a}, \tilde{b}]=\emptyset$. After defining $U$ to be zero outside $[a, b] \cup[\tilde{a}, \tilde{b}]$, formula (4) yields a selfsimilar solution that blows up as $t \rightarrow 1$. However, Lemma 1 will imply that this solution has at least one nonzero contact angle, and so we do not consider it here.

Let us return to the case in which $U$ 's maximal interval of positivity, $(a, b)$, is bounded and $0 \in(a, b)$. In this case, the resulting selfsimilar solution, $u$ is supported on an interval that shrinks in time. And so, just as one can use the translation invariance of the evolution equation (3) to construct a configuration of droplet steady states, one can construct a solution that blows up at time $T=1$ with the blowup centered at isolated points. Specifically, if $\left|x_{0}\right|>b-a$ then $u(x, t)+u\left(x-x_{0}, t\right)$ will be a solution that that blows up as $t \rightarrow 1$, with the blow up focused at the points $x=0$ and $x=x_{0}$. Proceeding in this way, one can construct infinitely many solutions that blow up at finite time, focusing at different points. This is a sufficiently natural construction that it will not considered further here.

With this understanding, in the following we use $\Omega$ interchangeably as the support of $U$ and as the closure of the maximal interval of positivity of a solution of the problem (12). We now prove some qualitative properties of selfsimilar solutions of the thin-film equation (3).

The following lemma is analogous to Lemma 2.5 in Beretta's paper [1], and we closely follow her proof.

Lemma 1. Let $U$ be a solution of the equation (12). If $0 \leq p<q$ are such that $(p, q)$ is contained in the support of $U, U(p)$ and $U(q)$ are both local minima or both local maxima then $U(p)>U(q)$. Similarly, if $q<p \leq 0$ and $U(p)$ and $U(q)$ are both local minima or both local maxima then $U(q)<U(p)$.

If $U$ is a zero-contact-angle solution with bounded support $[a, b]$ then $0 \in(a, b)$.

Proof. We first prove that $U(p)>U(q)$ when $0 \leq p<q$ are consecutive local minima of $U$. The theorem then follows immediately if $0 \leq p<q$ and $U(p)$ and $U(q)$ are 
local minima (possibly not consecutive). The case in which $0 \leq p<q$ and $U(p)$ and $U(q)$ are local maxima is treated in essentially the same manner. The cases in which $q<p \leq 0$ then follow from the reflection invariance of the equation.

Since $p<q$ are the locations of consecutive local minima, there exists a $r \in(p, q)$ such that $U(r)$ is a local maximum, $U$ is increasing on $(p, r)$ and $U$ is decreasing on $(r, q)$. We introduce

$$
F(x):=\gamma+\frac{H^{3}}{3}-\frac{1}{n+4} \int_{r}^{x} s U(s)^{1-n} d s
$$

Multiplying equation (13) by $U^{\prime}$,

$$
\frac{1}{2}\left(\left(U^{\prime}\right)^{2}\right)^{\prime}+\frac{1}{12}\left(U^{4}\right)^{\prime}=F(x) U^{\prime} .
$$

$U:[p, r] \rightarrow[U(p), U(r)]$ has an inverse, denoted $\nu^{-}$. Similarly, $\nu^{+}$is the inverse of $U$ on $[r, q]$.

Assume the lemma is not true: $U(p) \leq U(q)$. Take $\zeta \in[p, r)$ such that $U(\zeta)=$ $U(q)$. Integrating $(15)$ on the interval $(\zeta, q)$ and using that $F$ is a decreasing function on $[0, \infty)$ (since $p \geq 0$ ) yields:

$$
\begin{aligned}
0 \geq-\frac{1}{2} U^{\prime}(\zeta)^{2} & =\int_{\zeta}^{r} F(x) U^{\prime}(x) d x+\int_{r}^{q} F(x) U^{\prime}(x) d x \\
& =\int_{U(q)}^{U(r)} F\left(\nu^{-}(z)\right)-F\left(\nu^{+}(z)\right) d z>0 .
\end{aligned}
$$

This contradiction proves $U(p)>U(q)$, as desired.

It follows immediately that if $U$ is a zero-contact-angle solution with bounded support $[a, b]$ then $0 \in(a, b)$.

We see from equation (12) that the $n \leq 1$ and $n>1$ cases should be quite different. For example, if $n>1$ and $U \downarrow 0$ then $U^{\prime \prime \prime}$ diverges. This makes the analysis for the $n>1$ case harder, although it does ensure that all solutions $U$ have compact support:

Lemma 2. Assume that $n>1$ and $U$ is solution of equation (12). Let $\Omega$ be the support of $U$. Then $\Omega$ is bounded.

Proof. We first prove that if $x_{0} \in \operatorname{int}(\Omega)$ and $x_{0} \geq 0$ then $\Omega$ is bounded from above. The invariance of the equation (12) under the reflection about $x=0$ then yields that if $x_{0} \in \operatorname{int}(\Omega)$ and $x_{0} \leq 0$ then $\Omega$ is bounded from below. Combining these, $\Omega$ is bounded.

Let $x_{0} \in \operatorname{int}(\Omega), x_{0} \geq 0$. Assume $\Omega$ is not bounded from above. If $U(x) \leq U\left(x_{0}\right)$ for all $x>x_{0}$ then we use this upper bound in equation (13) to find

$U^{\prime \prime}(x)<\gamma+\frac{U\left(x_{0}\right)^{3}}{3}-\frac{1}{n+4} \int_{x_{0}}^{x} s U(s)^{1-n} d s<\gamma+\frac{U\left(x_{0}\right)^{3}}{3}-\frac{U\left(x_{0}\right)^{1-n}}{n+4} \frac{x^{2}-x_{0}^{2}}{2}$

for all $x \in\left(x_{0}, \infty\right)$. Therefore there exists $\tilde{x}>x_{0}$ such that $U^{\prime \prime}(x)<-2$ for all $x>\tilde{x}$, contradicting the nonnegativity of $U$. Thus it cannot be true that $U(x) \leq U\left(x_{0}\right)$ for all $x>x_{0}$. 
Therefore there exists $x_{1} \geq x_{0}$ such that $U\left(x_{1}\right)=U\left(x_{0}\right)$ and $U^{\prime}>0$ in a rightneighborhood of $x_{1}$. We claim there exists at an $x_{3}>x_{1}$ such that $U\left(x_{3}\right)$ is a local maximum. Assume not. Then $U$ is nondecreasing on $\left(x_{1}, \infty\right)$. If there exists $x_{2}>x_{1}$ such that $U\left(x_{2}\right) \geq \sqrt[3]{3 U^{\prime \prime}\left(x_{1}\right)+U\left(x_{1}\right)^{3}+3}$, we use this lower bound in equation (13) to find

$$
U^{\prime \prime}(x)<U^{\prime \prime}\left(x_{1}\right)+\frac{U\left(x_{1}\right)^{3}}{3}-\frac{U\left(x_{2}\right)^{3}}{3}<-1 \quad \forall x>x_{2} .
$$

Hence $U$ cannot be nondecreasing on $\left(x_{1}, \infty\right)$. If there is no such $x_{2}$, then $U(x)<$ $\sqrt[3]{3 U^{\prime \prime}\left(x_{1}\right)+U\left(x_{1}\right)^{3}+3}$ for all $x>x_{1}$ and we can use this upper bound in equation (13) to prove that $U^{\prime \prime}(x)<-1$ for all $x$ sufficiently large, again contradicting that $U$ is nondecreasing on $\left(x_{1}, \infty\right)$. Thus there exists $x_{3}>x_{1}$ such that $U\left(x_{3}\right)$ is a local maximum.

Since $U(x)<U\left(x_{3}\right)$ for all $x \in\left(x_{3}, \infty\right)$ by Lemma 1 , we have an upper bound for $U$ on $\left(x_{3}, \infty\right)$ and we can repeat the first argument in the proof, contradicting that $U$ is nonnegative. This proves that $\Omega$ is bounded above.

At this point, $U \in C^{1}(\Omega)$. We now prove that $U$ is $C^{2}$.

Lemma 3. Let $U$ be a solution of (12) with zero contact angles and let $\Omega=\left[x_{l}, x_{r}\right]$ be the support of $U$. Then $U \in C^{2}\left(\left[x_{l}, x_{r}\right]\right), U^{\prime \prime}\left(x_{l}\right) \geq 0$, and $U^{\prime \prime}\left(x_{r}\right) \geq 0$.

Proof. If $n \leq 1$ and $x \rightarrow \tilde{x}$ where $x \in \operatorname{int}(\Omega)$ and $\tilde{x}$ is on the contact line then $U^{\prime \prime}(x)$ will have a finite limit by equation (13) and $U \in C^{2}(\Omega)$. (Note that for $n \leq 1$ one does not need $U$ to have zero contact angles in order to ensure that $U \in C^{2}$.)

Now assume $n>1$. By Lemma 2, $U$ has compact support. Since $U$ has zero contact angles, $x_{l}<0<x_{r}$ (Lemma 1). By the zero-contact-angle assumption, $U^{\prime}(x) \rightarrow 0$ as $x \uparrow x_{r}$ and since we defined $U\left(x_{r}\right)$ to be zero, $U^{\prime}\left(x_{r}\right)$ exists and is equal to zero. Because $x_{r}>0$, equation (12) implies $U^{\prime \prime \prime}<0$ on $\left(x_{r}-\varepsilon, x_{r}\right)$ for some small positive $\varepsilon$. We now prove that $U^{\prime \prime}>0$ on this interval. Assume that $U^{\prime \prime}\left(x^{\prime}\right)<0$ for some $x^{\prime}$ in the interval. Then $U^{\prime \prime}<0$ on $\left(x^{\prime}, x_{r}\right)$ and hence $U^{\prime}$ is decreasing on $\left(x^{\prime}, x_{r}\right)$. Since $U\left(x^{\prime}\right)>0$ and $U\left(x_{r}\right)=0$ there exists a point $x^{\prime \prime}$ in between where $U^{\prime}\left(x^{\prime \prime}\right)<0$. But then $U^{\prime}<U^{\prime}\left(x^{\prime \prime}\right)$ on $\left(x^{\prime \prime}, x_{r}\right]$, contradicting the fact that $U^{\prime}\left(x_{r}\right)=0$. Thus $U^{\prime \prime}(x)$ is positive and decreasing once $x$ is sufficiently close to $x_{r}$, implying that $U^{\prime \prime}\left(x_{r}\right)$ exists and is nonnegative. By the invariance of the equation $U^{\prime \prime}\left(x_{l}\right)$ exists and is nonnegative. This proves $U \in C^{2}\left(\left[x_{l}, x_{r}\right]\right)$, as desired.

In fact, these arguments prove that if $\tilde{x} \neq 0$ is a touch-down point of $U$ and $U^{\prime}(x) \rightarrow 0$ as $x \rightarrow \tilde{x}$ then $U^{\prime \prime}(\tilde{x})$ is defined and finite. We use this observation to prove a non-existence result:

Theorem 4. If $n \geq 3 / 2$, then there are no zero-contact-angle solutions $U$ of equation (12).

Proof. Assume $U$ is a zero-contact-angle solution. By Lemma 3, $U \in C^{2}\left(\left[x_{l}, x_{r}\right]\right)$ where $\left[x_{l}, x_{r}\right]$ is the support of $U$ and $U^{\prime \prime}\left(x_{r}\right) \geq 0$. Since $U^{\prime}$ is bounded on $\left[x_{l}, x_{r}\right]$, equation (12) implies $U^{\prime \prime \prime} \rightarrow-\infty$ as $x \rightarrow x_{r}-$. Let $0<x<x_{r}$ be such that $U^{\prime \prime \prime}<0$ 
on $\left(x, x_{r}\right)$. Integrating equation (12),

$$
U^{\prime \prime}\left(x_{r}\right)-U^{\prime \prime}(x)=-\frac{1}{n+4} \int_{x}^{x_{r}} s U(s)^{1-n} d s-\frac{U\left(x_{r}\right)^{3}-U(x)^{3}}{3}
$$

which implies $\int_{x}^{x_{r}} s U(s)^{1-n} d s<\infty$. On the other hand, since $U^{\prime \prime}(x)>0, U^{\prime \prime}$ is decreasing on $\left(x, x_{r}\right)$ and $U\left(x_{r}\right)=U^{\prime}\left(x_{r}\right)=0$, if $s \in\left(x, x_{r}\right)$ then $U(s)<U^{\prime \prime}(x)(s-$ $\left.x_{r}\right)^{2} / 2$. And thus for $n \geq 3 / 2$,

$$
\infty>\int_{x}^{x_{r}} s U(s)^{1-n} d s \geq\left(\frac{U^{\prime \prime}(x)}{2}\right)^{1-n} \int_{x}^{x_{r}} s\left(s-x_{r}\right)^{2-2 n} d s=\infty .
$$

This is impossible, proving that $U$ does not exist.

Remark. Note that the reasons for the nonexistence result of the Theorem are localized near the contact line. Furthermore, note that only the first of two terms on the righthand side of (12) was relevant for the argument. A similar term, of the same sign, appears in the equation for the receding traveling fronts $\left(c U^{\prime \prime \prime}=U^{1-n}, c<0\right)$ of the thin-film equation equation $\left(u_{t}=-\left(u^{n} u_{x x x}\right)_{x}\right)$. Therefore it is not surprising that zero-contact-angle receding traveling fronts do not exist when $n \geq 3 / 2$. What is more, Beretta, Bertsch, and Dal Passo [2] proved that if $n \geq 3 / 2$ and $u$ is a solution of $u_{t}=-\left(u^{n} u_{x x x}\right)_{x}$, then the support of $u$ cannot contract as time passes.

We now present a comparison result relating the selfsimilar profiles $U$ to steady states $V$.

Lemma 5. Let $U$ solve (12) with $x_{0} \geq 0, U\left(x_{0}\right)=H, U^{\prime}\left(x_{0}\right)=\xi$ and $U^{\prime \prime}\left(x_{0}\right)=\gamma_{1}$ and $V$ solve (6) with $V\left(x_{0}\right)=U\left(x_{0}\right), V^{\prime}\left(x_{0}\right)=U^{\prime}\left(x_{0}\right)$ and $V^{\prime \prime}\left(x_{0}\right)=\gamma_{2} \geq \gamma_{1}$. Assume either $\xi<0$ or $\xi=0$ and $\gamma_{2} \leq 0$. Let

$$
X:=\sup \left\{x \mid U^{\prime}<0 \text { and } U>0 \text { on }\left(x_{0}, x\right)\right\} .
$$

Then for all $x \in\left(x_{0}, X\right), U(x)<V(x)$. Furthermore, let $\nu$ be the inverse of $U$ restricted to $\left[x_{0}, X\right]$. Then for all $y \in\left[V_{\text {min }}, H\right), U^{\prime}(\nu(y))<p(y)$, where $p$ is defined by (9).

Proof. For $y \in[U(X), H]$ define

$$
q(y)=U^{\prime}(\nu(y)) \quad \text { and } \quad G(y)=\frac{1}{n+4} \int_{x_{0}}^{\nu(y)} s U(s)^{1-n} d s>0 .
$$

The integral form of the equation can then be written as

$$
q(y) q^{\prime}(y)=\gamma+\frac{H^{3}}{3}-\frac{y^{3}}{3}-G(y), \quad q(H)=\xi .
$$

Therefore using (9), for $y \in[U(X), H) \cap\left[V_{\text {min }}, H\right)$

$q(y)=-\frac{1}{\sqrt{6}} \sqrt{6 \xi^{2}+y\left(H^{3}-y^{3}\right)-3\left(4 \gamma_{1}+H^{3}\right)(H-y)+12 \int_{y}^{H} G(z) d z}<p(y)$.

It follows that $U(X) \leq V_{\min }$, proving the second claim of the lemma. The first claim now easily follows. 


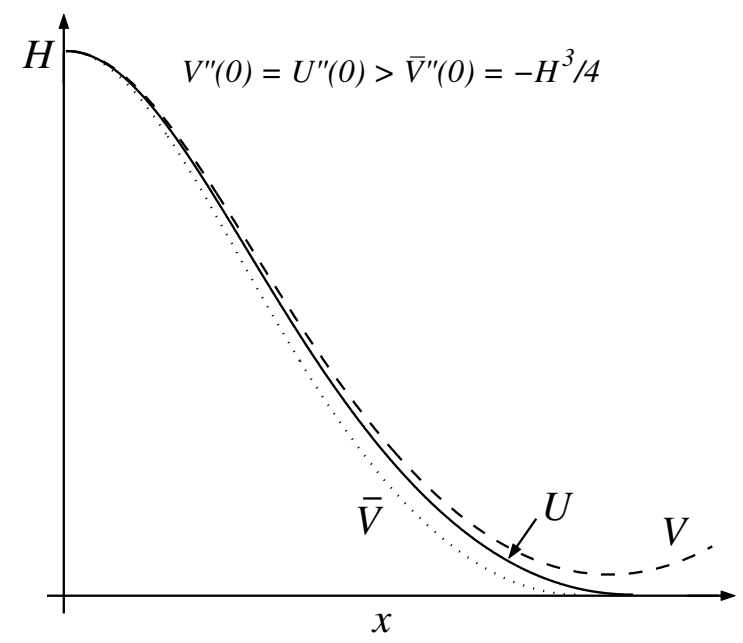

Figure 1: An illustration of comparison results of Lemmas 5 and 7. The initial data for $U$ are such that it touches down with zero contact angle. $V>U$ by Lemma 5 , while $U>\bar{V}$ by Lemma 7 .

Recall that if $x_{0}=\xi=0$ and $V^{\prime \prime}(0)=-H^{3} / 4$ then $V$, the solution of (6), has compact support and zero contact angle and is denoted $\bar{V}$. We use Lemma 5 to find a comparison result in which $U$ is bounded by $\bar{V}$ :

Corollary 6. Let $U$ be a solution of (12) with support $\left[x_{l}, x_{r}\right]$. Assume that $U$ has a local maximum at $x_{0}$ and $U^{\prime \prime}\left(x_{0}\right) \leq-H^{3} / 4$. If $x_{0} \geq 0$ then $U$ is decreasing on $\left(x_{0}, x_{r}\right)$ and $\lim \sup _{x \rightarrow x_{r}^{-}} U^{\prime}(x)<0$. If $x_{0} \leq 0$ then $U$ is increasing on $\left(x_{l}, x_{0}\right)$ and $\liminf _{x \rightarrow x_{l}^{+}} U^{\prime}(x)>0$.

We now give a comparison result in which $\bar{V}$ is bounded by $U$.

Lemma 7. Let $U$ be a solution of (12) that touches down with zero contact angles at $x= \pm L$ and has a global maximum at $x_{0}=0 . \bar{V}$ and $\bar{p}$ are defined above, after equation (10) with $H=U(0)$.

a) $U$ is invertible on intervals $\left[x_{0}, x_{1}\right],\left[x_{1}, x_{2}\right], \ldots\left[x_{k-1}, x_{k}\right]$ where $x_{k}=L$ and $U^{\prime}\left(x_{j}\right)=0$ for $j=0 . . k$. Let $\nu_{j}, j=1 \ldots k$, be the respective inverses. Then $U^{\prime}\left(\nu_{j}(y)\right)>\bar{p}(y)$ for $y \in\left(U\left(x_{j-1}\right), U\left(x_{j}\right)\right)$ for $j=1 \ldots k$.

b) ${ }^{1} U>\bar{V}$ on $(0, L)$ and $m=2 \int_{0}^{L} U(x) d x>2 \pi \sqrt{2 / 3}$.

\footnotetext{
${ }^{1}$ Although in the paper [1] it is claimed that there exist selfsimilar spreading (i.e. source-type) solutions of the thin-film equation (3) of any mass, Witelski, Bernoff, and Bertozzi [25] noted that that is not the case. In fact for the ODE that describes the profile of the selfsimilar spreading solutions a claim analogous to this one can be shown. Only that in that case $U<\bar{V}$, which implies that the mass of the solutions is less then the critical mass $2 \pi \sqrt{2 / 3}$.
} 
Proof. Proof of claim a). First consider the interval $\left[x_{0}, x_{1}\right]$. Let $q(y)=U^{\prime}\left(\nu_{1}(y)\right)$ and $H=U(0)$. Because $U$ has zero contact angles, $\gamma=U^{\prime \prime}(0)>-H^{3} / 4$ by Corollary 6 . Since $\bar{V}^{\prime \prime}(0)=-H^{3} / 4$, there exists some $\varepsilon>0$ such that $q(y)>\bar{p}(y)$ on $(H-\varepsilon, H)$. Assume $q>\bar{p}$ is not true on $\left(U\left(x_{1}\right), U\left(x_{0}\right)\right)$. Let $Y$ be the largest $y \in\left(U\left(x_{1}\right), U\left(x_{0}\right)\right)$ such that $q(y)=\bar{p}(y)$. Then $q^{\prime}(Y) \geq \bar{p}^{\prime}(Y)$ and hence $U^{\prime \prime}\left(\nu_{1}(Y)\right) \leq \bar{V}^{\prime \prime}\left(\bar{V}^{-1}(Y)\right)$. Let $\tilde{x}=\nu_{1}(Y)$. We use $\tilde{x}$ to shift $\bar{V}$, introducing $V(x)=\bar{V}\left(x-\tilde{x}+\bar{V}^{-1}(Y)\right)$. $V$ satisfies equation (6) with initial data $V(\tilde{x})=U(\tilde{x}), V^{\prime}(\tilde{x})=U^{\prime}(\tilde{x})$ and $V^{\prime \prime}(\tilde{x}) \geq$ $U^{\prime \prime}(\tilde{x})$. Since $\tilde{x}>0$, Lemma 5 (with $p=\bar{p}$ ) implies that $q(y)<\bar{p}(y)$ for $y \in$ $\left[U\left(x_{1}\right), U\left(x_{0}\right)\right)$ and thus $0=q\left(U\left(x_{1}\right)\right)<\bar{p}\left(U\left(x_{1}\right)\right) \leq 0$. This is impossible and hence $q>\bar{p}$ on $\left(U\left(x_{1}\right), U\left(x_{0}\right)\right)$ as desired. Now consider the interval $\left[x_{j-1}, x_{j}\right]$ where $2 \leq j \leq k$. If $U$ is increasing on the interval, the result holds immediately because $\bar{p}(y) \leq 0$ for $y \in[0, H]$. Assume $U$ is decreasing and let $q(y)=U^{\prime}\left(\nu_{j}(y)\right)$. By assumption, $q\left(U\left(x_{j-1}\right)\right)=0$ and $\bar{p}\left(U\left(x_{j-1}\right)\right)<0$, hence there exists some $\varepsilon>0$ such that $q(y)>\bar{p}(y)$ on $\left(U\left(x_{j-1}\right)-\varepsilon, U\left(x_{j-1}\right)\right)$. Arguing as above, $q>\bar{p}$ on $\left(U\left(x_{j}\right), U\left(x_{j-1}\right)\right)$ as desired.

Proof of claim b). Given $y \in[0, H)$, let $0<\tilde{x}_{1}<\cdots<\tilde{x}_{s} \leq L$ be all those points that satisfy $U\left(\tilde{x}_{j}\right)=y$. Let $\eta$ be the inverse of $\bar{V}$. Using part a) and the fundamental theorem of calculus, one argues that $\eta(y)<\tilde{x}_{1}$. This implies $U>\bar{V}$ on $(0, L)$, as desired. The mass statement then follows because $U$ is symmetric about $x=0$.

The methods just used also yield a more general comparison result:

Corollary 8. Let $U$ be a zero-contact-angle solution of (12) supported on $\left(x_{l}, x_{r}\right)$. Assume $U(\hat{x})$ is a global maximum. Let $\bar{V}$ be the zero-contact-angle solution of $(6)$ with $\bar{V}(0)=H:=U(\hat{x})$. Then $U(x)>\bar{V}(x-\hat{x})$ for all $x \in\left(x_{l}, \hat{x}\right) \cup\left(\hat{x}, x_{r}\right)$ and $m=\int_{x_{l}}^{x_{r}} U(x) d x>M_{c}$.

The fact that $m>M_{c}=2 \pi \sqrt{2 / 3}$ is not surprising because, as explained after the equation (3), it is known that the solutions of mass less than $M_{c}$ do not blow up [25].

Proof. If $\hat{x}=0$, then the result is simply Lemma 7. Since the equation (12) is invariant under reflection about $x=0$, we can assume that $\hat{x}<0$. Let $V(x)=\bar{V}(x-\hat{x})$. To establish the claim for all $x_{l}<x<\hat{x}$, reflect $U$ about $x=0$ so that $\hat{x}>0$, define $x_{0}=\hat{x}$, and repeat the arguments in the proof of Lemma 7. The $\hat{x}<x<x_{r}$ case remains. Lemma 1 implies that since $\hat{x}$ is the location of a global maximum it must also be the location of the right-most local maximum on $\left(x_{l}, 0\right]$.

Let $\left[\hat{x}, x_{1}\right],\left[x_{1}, x_{2}\right], \ldots,\left[x_{k-1}, x_{k}\right]$ where $x_{k}=x_{r}$ be the maximal intervals where $U$ is invertible. Consider first the interval $\left[\hat{x}, x_{1}\right]$. Let $\nu$ be the inverse of $U$ on the interval, let $q(y)=U^{\prime}(\nu(y))$ and let $G$ be as in Lemma 5. Let $V^{-1}:[0, H] \rightarrow$ $\left[\hat{x}, \hat{x}+\bar{x}_{\text {min }}\right]$, resulting in $p(y)=V^{\prime}\left(V^{-1}(y)\right)=\bar{p}(y)$.

Case $1^{\circ}$ If $x_{1} \leq 0$. Then $G(y)<0$ on $\left[U\left(x_{1}\right), H\right)$. Thus, arguing as in the proof of Lemma 5, yields that $q(y)>p(y)$ for $y \in\left[U\left(x_{1}\right), H\right)$. Thus $U>V$ on $\left(\hat{x}, x_{1}\right]$. The analysis on $\left[x_{1}, x_{2}\right]$ is trivial since $U$ is nondecreasing on the interval while $\bar{V}$ is decreasing. The proof of the claim on the remaining intervals reduces to the one given in Lemma 7. 
Case $2^{\circ}$ If $x_{1}>0$. Arguing as above we obtain that $q(y)>p(y)$ on $y \in$ $(U(0), H)$. Since $q(U(0))>p(U(0))$ the argument on $\left[0, x_{1}\right]$ reduces to the case treated in Lemma 7. So does the argument on the rest of the intervals.

We close this section with a nonexistence result that partially complements Theorem 4. Specifically, for $n \in(0,3 / 2)$ it is not possible to find selfsimilar blow-up solutions of the thin-film equation (3) that have zero contact angles if they are not "sufficiently large" one time unit before blow up:

Theorem 9. Assume $0<n<3 / 2$. There are no zero-contact-angle solutions of (12) that have $\max \{U\}<\max \left\{1,(3-2 n)^{-2 / 11}\right\}$.

Proof. Let $U$ be a zero-contact-angle solution of (12) with maximum $H$ achieved at $x=x_{0}$. Because of the symmetry of the equation, we can assume that $x_{0} \geq 0$. Let $\left[x_{l}, x_{r}\right]$ be the support of $U$. Since $U$ has a maximum at $x_{0}, \gamma:=U^{\prime \prime}\left(x_{0}\right) \leq 0$. By Lemma $3,0 \leq U^{\prime \prime}\left(x_{r}\right)<\infty$. From equation (13),

$$
\frac{H^{3}}{3} \geq-U^{\prime \prime}\left(x_{r}\right)+\gamma+\frac{H^{3}}{3}=\frac{1}{n+4} \int_{x_{0}}^{x_{r}} s U(s)^{1-n} d s
$$

Case $1^{\circ} n \in(0,1)$. By Lemma 7 follows that $U>\bar{V}\left(\cdot-x_{0}\right)$ on $\left(x_{0}, x_{0}+\bar{x}_{\min }\right)$, and we know that $\bar{V}(x)>H-\frac{H^{3}}{8} x^{2}$. Therefore

$$
\int_{x_{0}}^{x_{r}} s U(s)^{1-n} d s \geq \int_{0}^{\sqrt{8} / H}\left(s+x_{0}\right)\left(H-\frac{H^{3}}{8} s^{2}\right)^{1-n} d s \geq \frac{4 H^{-1-n}}{2-n} .
$$

Hence

$$
\frac{H^{3}}{3} \geq \frac{1}{n+4} \frac{4 H^{-1-n}}{2-n} \Longrightarrow H^{n+4}>1 \Longrightarrow H>\max \left\{1,(3-2 n)^{-2 / 11}\right\} .
$$

Case $2^{\circ} \quad n \in[1,3 / 2)$. From equation (13), $U^{\prime \prime}(x) \leq H^{3} / 3$ for $x \in\left[x_{0}, x_{r}\right]$. Therefore $U(x) \leq\left(x-x_{r}\right)^{2} H^{3} / 6$ and hence

$$
\begin{aligned}
\int_{x_{0}}^{x_{r}} s U(s)^{1-n} d s & \geq \frac{H^{3-3 n}}{6^{1-n}} \int_{x_{0}}^{x_{r}} s\left(s-x_{r}\right)^{2-2 n} d s \\
& \geq \frac{H^{3-3 n}}{2} \cdot \frac{\left(x_{r}-x_{0}\right)^{4-2 n}}{3-2 n} \geq \frac{2 H^{-n-1}}{3-2 n}
\end{aligned}
$$

where in the last inequality we used $x_{r}-x_{0}>\bar{x}_{\min }>5.9 / H$ (by Lemma 7 and (11)). It follows that $H^{4+n}>\frac{1}{3-2 n}$, and hence $H>\max \left\{1,(3-2 n)^{-2 / 11}\right\}$.

\section{EXISTENCE OF ZERO-CONTACT-ANGLE SOLUTIONS}

In this section, we prove that if $0<n<3 / 2$, equation (12) has a zero-contactangle solution, $U$, that has compact support $[-L, L]$, is symmetric about $x=0$, and is decreasing on $[0, L]$. Recall that the steady state with $\bar{V}(0)=H, \bar{V}^{\prime}(0)=0$, and $\bar{V}^{\prime \prime}(0)=-H^{3} / 4$ has compact support and zero contact angles. To prove the existence of the desired $U$, we first prove that if $U(0)=H, U^{\prime}(0)=0$, and $U^{\prime \prime}(0)$ is "close" to $-H^{3} / 4$ and $V$ is the steady state with the same initial data as $U$ then if $H$ is "large" 
this will ensure $U$ and $V$ are "close" for the first downstroke of $U$. We then use this information in a shooting argument to prove the existence of the desired $U$. We carry out the argument for explicit range of $H$ and thus our arguments involve a number of explicit constants. The reader who would like to only understand the gist of the argument can read the proofs without going through explicit computations and only assume that $H$ is very large.

4.1. The $n \in(0,1]$ Case. We first prove that the steady state $V$ controls $U$ for the first downstroke of $U$ :

Lemma 10. Let $0<n \leq 1$. Assume $U(0)=V(0)=H, U^{\prime}(0)=V^{\prime}(0)=0$, and $U^{\prime \prime}(0)=V^{\prime \prime}(0)=\gamma \in\left[-H^{3} / 4,-H^{3} / 4+3 H\right]$. Let $U$ and $V$ be the resulting solutions of equations (12) and (6) respectively. If $\gamma>-H^{3} / 4$ then $V$ is defined on $\mathbb{R}$. If $\gamma=-H^{3} / 4$ then $V$ is taken to be extended to $\mathbb{R}$ via formula (10). Define

$$
X:=\sup \left\{x>0 \mid U^{\prime}<0 \text { and } U>0 \text { on }(0, x)\right\} .
$$

If $H \geq 40$ then $X<6.2 / H$ and for all $x \in[0, X]$

$$
|U(x)-V(x)|<\frac{27}{H},\left|U^{\prime}(x)-V^{\prime}(x)\right|<9,\left|U^{\prime \prime}(x)-V^{\prime \prime}(x)\right|<1.3 H .
$$

Proof. We reintroduce the notation $x_{0} \geq 0$ for the point at which the initial data are specified, since that will be useful in Section 5. Introducing $W=U-V$, note that

$$
W^{\prime \prime \prime}(x)=-\frac{x}{n+4} U^{1-n}(x)-(U(x)+V(x)) V^{\prime}(x) W(x)-U^{2}(x) W^{\prime}(x) .
$$

Let $g(x):=\max \left\{\left|W^{\prime \prime}(s)\right| \mid s \in\left[x_{0}, x\right]\right\} . g$ is absolutely continuous and nondecreasing, $\left|W^{\prime}(x)\right| \leq g(x)\left(x-x_{0}\right)$, and $|W(x)| \leq g(x)\left(x-x_{0}\right)^{2} / 2$. Hence

$$
g^{\prime}(x) \leq \frac{x U^{1-n}(x)}{n+4}+(U(x)+V(x))\left|V^{\prime}(x)\right| \frac{\left(x-x_{0}\right)^{2}}{2} g(x)+U^{2}(x)\left(x-x_{0}\right) g(x)
$$

at almost all $x$ in the domain of $U$. (The domain of $g$ equals the domain of $U$ by Lemma 5.) Lemma 5 also implies $U(x) \leq V(x)$ for $x \in\left[x_{0}, X\right]$. Therefore

$$
g^{\prime}(x) \leq \frac{x}{n+4} U^{1-n}(x)+\left[\left(x-x_{0}\right)^{2} V(x)\left|V^{\prime}(x)\right|+\left(x-x_{0}\right) V^{2}(x)\right] g(x)
$$

and hence

$$
g(x) \leq e^{\int_{x_{0}}^{x}\left(s-x_{0}\right)^{2} V(s)\left|V^{\prime}(s)\right|+\left(s-x_{0}\right) V^{2}(s) d s} \int_{x_{0}}^{x} \frac{s U^{1-n}(s)}{n+4} d s .
$$

Here, we used a Grönwall inequality: Let $\phi$ and $\psi$ be nonnegative, continuous functions. If

$$
G^{\prime}(x) \leq \phi(x)+\psi(x) G(x) \quad \text { for all } x \geq a
$$

then for all $x>a$

$$
G(x) \leq e^{\int_{a}^{x} \psi(s) d s} G(a)+\int_{a}^{x} \phi(s) e^{\int_{s}^{x} \psi(z) d z} d s \leq e^{\int_{a}^{x} \psi(s) d s}\left(G(a)+\int_{a}^{x} \phi(s) d s\right) .
$$


We now go back to $x_{0}=0$ and introduce $x_{1}:=\min \left\{\frac{6.2}{H}, X\right\}$. Let $x_{\text {min }}$ be, as before, the location of the first positive minimum of $V ; V^{\prime}\left(x_{\text {min }}\right)=0$ and $V^{\prime}<0$ on $\left(0, x_{\min }\right)$. To bound $g(x)$ on $\left[0, x_{1}\right]$ we estimate

$I=\int_{0}^{x_{\text {min }}} s^{2} V(s)\left|V^{\prime}(s)\right|+s V^{2}(s) d s \quad$ and $\quad I I=\int_{x_{\text {min }}}^{x_{1}} s^{2} V(s)\left|V^{\prime}(s)\right|+s V^{2}(s) d s$. Integration by parts yields

$$
I \leq 2 \int_{0}^{x_{\min }} s V^{2}(s) d s
$$

Let $\hat{\gamma}=\left(\gamma+H^{3} / 4\right) / H^{3}$. By property (P1) in $\S 2, V_{\min }=V\left(x_{\min }\right)<12 \hat{\gamma} H$. Also since $V$ satisfies (7) for all $s, V^{\prime \prime}(s) \leq H^{3}(1 / 12+\hat{\gamma})$. Therefore, since $\hat{\gamma}<1 / 500$,

$$
V(x) \leq \min \left\{H, 12 \hat{\gamma} H+\frac{H^{3}}{23}\left(x_{\text {min }}-x\right)^{2}\right\} \quad \forall x .
$$

Using this estimate and $x_{\min }<5.95 / H$ (Property (P3) in $\S 2$ ), one finds

$$
I \leq 2 \int_{0}^{x_{\min }} s V^{2}(s) d s<5.8 .
$$

We now estimate $I I$. If $x_{1} \leq x_{\text {min }}$ then $I I \leq 0$, so let us assume $x_{1}>x_{\text {min }}$. Since $x_{1}<2 x_{\min }, V^{\prime}>0$ on $\left(x_{\min }, x_{1}\right)$. Hence using (20) along with the fact that $x_{1}-x_{\min }<0.4 / H$ we obtain

$$
I I=\frac{1}{2} \int_{x_{\text {min }}}^{x_{1}}\left(s^{2} V^{2}(s)\right)^{\prime} d s \leq \frac{1}{2} x_{1}^{2} V^{2}\left(x_{1}\right)<0.02 .
$$

Therefore

$$
\int_{0}^{x_{1}} s^{2} V(s)\left|V^{\prime}(s)\right|+s V^{2}(s) d s=I+I I<5.9
$$

and the Grönwall inequality (18) imply

$$
g\left(x_{1}\right) \leq e^{6} \frac{1}{4} H^{1-n} \frac{x_{1}^{2}}{2}<\frac{(6.2)^{2} e^{6}}{8} H^{-1-n}<1.3 H
$$

Since $g$ is nondecreasing function, for all $x \leq x_{1}$

$$
\left|U^{\prime \prime}(x)-V^{\prime \prime}(x)\right|<1.3 H \Longrightarrow\left|U^{\prime}(x)-V^{\prime}(x)\right|<9 \text { and }|U(x)-V(x)|<\frac{27}{H} .
$$

The second two inequalities were found by integrating and using $x \leq x_{1} \leq 6.2 / H$.

We finish by proving that $x_{1}=X$, that is $X<6.2 / H$. Assume not; assume $X \geq 6.2 / H$. Therefore $x_{1}=6.2 / H$ and $U^{\prime} \leq 0$ on $\left[0, x_{1}\right]$. Let $x_{2}$ be the lesser of $6.2 / H$ and the smallest $x>x_{\min }$ for which $V(x)=3$. If $V\left(x_{2}\right)=3$ then $V^{\prime}\left(x_{2}\right)=$ $-p(3)>10$ and therefore $\left|V^{\prime}\left(x_{2}\right)-U^{\prime}\left(x_{2}\right)\right|>10$, contradicting the estimate (16). Hence $x_{2}=6.2 / H$ and $V<3$ on $\left(x_{\min }, 6.2 / H\right)$. By equation (7), $V^{\prime \prime}(x)>H^{3} / 12-$ $27 / 3>H^{3} / 13$ for $x \in\left(x_{\min }, 6.2 / H\right)$. Finally, $6.2 / H-x_{\min }>0.25 / H$ and hence $V^{\prime}(6.2 / H)>0.01 H^{2}>10$, which leads to a contradiction as before. This proves that $x_{1}=X$ and hence the bounds (16) hold on $[0, X]$, as desired.

We now prove the existence result: 
Theorem 11. Assume $0<n \leq 1$. Given $H \geq 40$, there exists $\gamma \in\left[-H^{3} / 4,-H^{3} / 4+\right.$ $3 H]$ such that the solution of (12) with $U(0)=H, U^{\prime}(0)=0$, and $U^{\prime \prime}(0)=\gamma$ has compact support $[-L, L], U^{\prime}( \pm L)=0$, and $U$ is decreasing on $[0, L]$.

This is proven using a shooting argument. Let $U$ be the solution of equation (12) with initial data $U(0)=H, U^{\prime}(0)=0$ and $U^{\prime \prime}(0)=\gamma<0$. We define

$$
\begin{aligned}
& \alpha(H, \gamma):=\min \left\{x>0 \mid U^{\prime}(x)=0 \text { or } x=\infty\right\} \\
& \beta(H, \gamma):=\min \left\{x>0 \mid \lim _{s \rightarrow x^{-}} U(s)=0 \text { or } x=\infty\right\} \\
& X(H, \gamma):=\min \{\alpha(H, \gamma), \beta(H, \gamma)\}
\end{aligned}
$$

We also define

$$
\begin{aligned}
& S^{+}(H)=\left\{\gamma \in \mathbb{R}^{-} \mid \alpha(H, \gamma) \leq \beta(H, \gamma)\right\} \\
& S^{-}(H)=\left\{\gamma \in \mathbb{R}^{-} \mid \alpha(H, \gamma) \geq \beta(H, \gamma)\right\} .
\end{aligned}
$$

By construction, $S^{-}(H) \cup S^{+}(H)=\mathbb{R}^{-}$. If there exists $\gamma \in S^{-}(H) \cap S^{+}(H)$ and $X(H, \gamma)$ is finite then the desired solution exists.

Proof. Let $I=\left[-H^{3} / 4,-H^{3} / 4+3 H\right], \widetilde{S}^{+}=S^{+}(H) \cap I$, and $\widetilde{S}^{-}=S^{-}(H) \cap I$. Since $X(H, \gamma)$ is finite for $\gamma \in I$ by Lemma 10 , it suffices to show that $\widetilde{S}^{+} \cap \widetilde{S}^{-} \neq \emptyset$. We first prove that $\widetilde{S}^{-}$and $\widetilde{S}^{+}$are both nonempty. By Corollary $6,-H^{3} / 4 \in \widetilde{S}^{-} \backslash \widetilde{S}^{+}$. We now prove that $-H^{3} / 4+3 H \in \widetilde{S}^{+}$. Let $U$ and $V$ be the solutions of (12) and (6) with $\gamma=-H^{3} / 4+3 H$. Then by property (P1) in $\S 2 V_{\min }>30 / H$. If $\gamma \in \widetilde{S}^{-}$ then $X=\beta(H, \gamma)$ and by bound (16) $|U(X)-V(X)|<27 / H$. But $U(X)=0$ (since $\gamma \in \widetilde{S}^{-}$), contradicting the bound (16). Thus $-H^{3} / 4+3 H \notin \widetilde{S}^{-}$hence $-H^{3} / 4+3 H \in$ $\widetilde{S}^{+}$, as desired.

Since $n \leq 1$ and $\alpha(=X)$ is uniformly bounded for $\gamma \in \widetilde{S}^{+}$by Lemma 10 , the continuous dependence on initial data implies that $\widetilde{S}^{+}$is closed in $I$. (Recall that for $n \leq 1$ the ODE (12) is not singular at $U=0$.) We now prove that if $\widetilde{S}^{+} \cap \widetilde{S}^{-}=\emptyset$ then $\widetilde{S}^{+}$is open in $I$. Since $I$ is connected and $\widetilde{S}^{+}$is nonempty, it would then follow that $\widetilde{S}^{+}=I$ which is impossible because $-H^{3} / 4 \notin \widetilde{S}^{+}$. Therefore $\widetilde{S}^{+} \cap \widetilde{S}^{-} \neq \emptyset$ and the desired solution exists.

Proving that if $\widetilde{S}^{+} \cap \widetilde{S}^{-}=\emptyset$ then $\widetilde{S}^{+}$is open uses Lemma 10. Indeed, if $H$ is not sufficiently large, it is possible for $\widetilde{S}^{+}$to not be open; see Figure 2. Assume that $\widetilde{S}^{+} \cap \widetilde{S}^{-}=\emptyset$. Let $\gamma \in \widetilde{S}^{+}$, and $U$ be the solution of equation (12) with $U(0)=H$, $U^{\prime}(0)=0$, and $U^{\prime \prime}(0)=\gamma$. Then $U(X)>0$ and, by Lemma $5, V_{\min }>U(X)$. By estimate (16), $V(X)-V_{\text {min }}<V(X)-U(X)<27 / H$. Since $\gamma \in I, V_{\min }<$ $36 / H$. Therefore $V(X)<63 / H$. By equation (6), it follows $V^{\prime \prime}(X)>H^{3} / 12-$ $(63 / H)^{3} 1 / 3>3 H$. Estimate (16) then implies $U^{\prime \prime}(X)>H>0$. So for $x$ slightly larger than $X, U^{\prime}(x)>0$. Continuous dependence on initial data now implies that $\widetilde{S}^{+}$ is open in $I$, as desired.

Now that we have proven the existence of a selfsimilar compactly supported solution with zero contact angles that is symmetric about $x=0$, we wish to prove that 

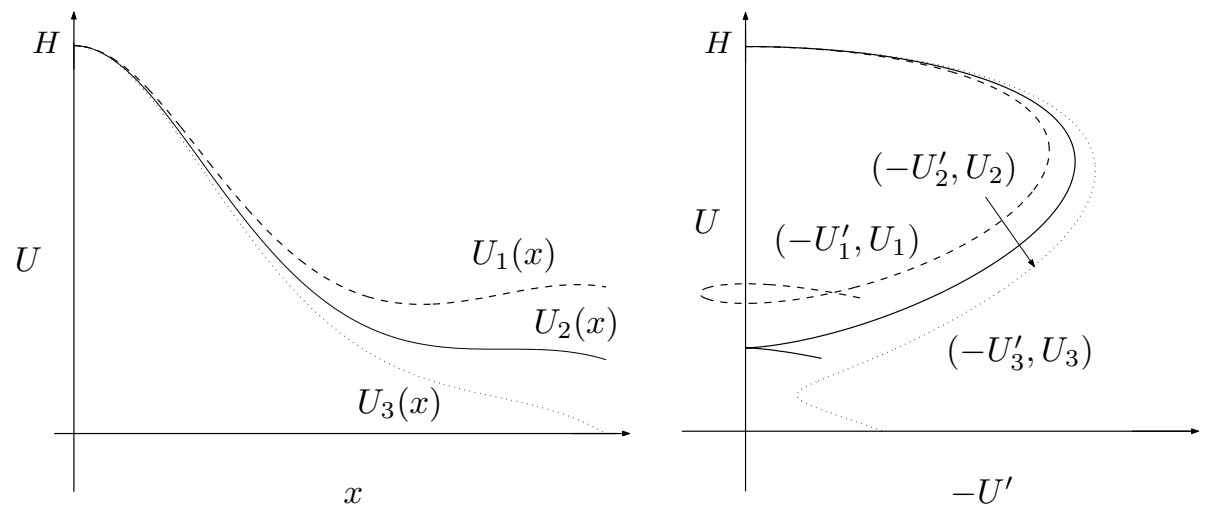

Figure 2: An illustration of how, when $H$ is small, $S^{+}(H)$ can fail to be open. On the left, we plot solutions $U$ versus $x$. All three solutions have $U(0)=H$ and $U^{\prime}(0)=0$ but the values of $U^{\prime \prime}(0)$ vary. On the right, we present a phase-plane plot, plotting $-U^{\prime}$ versus $U$. $U_{1}$ has two critical points, $U_{2}$ has one, and $U_{3}$ has none. Hence for small $\varepsilon>0,\left(U_{2}^{\prime \prime}(0)-\varepsilon, U_{2}^{\prime \prime}(0)\right) \subset S^{-}(H)$ and $\left[U_{2}^{\prime \prime}(0), U_{2}^{\prime \prime}(0)+\varepsilon\right) \subset S^{+}(H)$ and $S^{+}(H)$ is not open.

the larger $H$ is, closer the profile $U$ is to the steady state profile $\bar{V}$. To do this, we first introduce a weighted $C^{2}$-norm:

$$
\|U\|_{[x, y]}:=\max \left\{(y-x)^{2}\left\|U^{\prime \prime}\right\|_{L^{\infty}[x, y]},(y-x)\left\|U^{\prime}\right\|_{L^{\infty}[x, y]},\|U\|_{L^{\infty}[x, y]}\right\} .
$$

We first prove that steady states are close to one another with respect to this norm:

Lemma 12. Let $H>40, a>1$ and $0<\varepsilon<1$. Let $\delta=\varepsilon e^{-6} / 40$ and let $V$ be the solution of (6) with initial data $V(0)=H, V^{\prime}(0)=0$ and $V^{\prime \prime}(0)=\gamma \in$ $\left(-H^{3} / 4,-H^{3} / 4+\delta H^{-a}\right)$. Let $\bar{V}$ be the solution of (6) with initial data $\bar{V}(0)=H$, $\bar{V}^{\prime}(0)=0$, and $\bar{V}^{\prime \prime}(0)=-H^{3} / 4$. Then $\|V-\bar{V}\|_{[0,6.2 / H]}<\varepsilon H^{-a-2}$.

By property (P3), $\bar{V}$ is supported on $\left[-\bar{x}_{\text {min }}, \bar{x}_{\text {min }}\right]$ where $\bar{x}_{\text {min }}<5.95 / H$. In Lemma 12, Lemma 13, and Corollary 14, the weighted norm is referring to a longer interval than the support of $\bar{V}$. To do this, we assume that $\bar{V}$ has been continued by (10) beyond its support (basically by reflection about $x=\bar{x}_{\text {min }}$ ).

Proof. Note that $\delta H^{-a}<3 H$ and hence by property (P3) $x_{\text {min }}<\bar{x}_{\text {min }}$. From (9) it follows that $V \geq \bar{V}$ on $\left[0, \bar{x}_{\min }\right] . V$ is symmetric about $x=x_{\min }$ and $\bar{V}$ is (after extension) symmetric about $\bar{x}_{\text {min }}$, and so it follows that $V \geq \bar{V}$ on $\left[0,2 x_{\min }\right]$. To estimate the difference between $V$ and $\bar{V}$ we use the approach of Lemma 10. Let $W=$ $V-\bar{V}$ and $g(x):=\max \left\{\left|W^{\prime \prime}(s)\right| \mid s \in[0, x]\right\}$. Arguing as in the proof of Lemma 10 one obtains

$$
g^{\prime}(x) \leq(V(x)+\bar{V}(x))\left|V^{\prime}(x)\right| \frac{x^{2}}{2} g(x)+\bar{V}^{2}(x) x g(x)
$$


Using that $\bar{V} \leq V$ and the Grönwall inequality (19) yields:

$$
g(x) \leq e^{\int_{0}^{x} s^{2} V(s)\left|V^{\prime}(s)\right|+s V^{2}(s) d s} \delta H^{-a}
$$

The estimate (21) implies that $g(x)<e^{6} \delta H^{-a}$ for all $x \in[0,6.2 / H] \subset\left[0,2 x_{\min }\right]$. The claim now follows from the definitions of $g$ and $\delta$ by integrating in $x$.

We now prove that the selfsimilar profile $U$ is close to the compactly supported zero-contact-angle steady state $\bar{V}$ with respect to the weighted $C^{2}$-norm:

Lemma 13. Let $H>100$, and $\gamma \in\left[-H^{3} / 4,-H^{3} / 4+3 H\right]$ such that the solution of (12) with $U(0)=H, U^{\prime}(0)=0$ and $U^{\prime \prime}(0)=\gamma$ touches down at $L$ with zero contact angle. Let $\bar{V}$ be the solution of (6) with $\bar{V}(0)=H, \bar{V}^{\prime}(0)=0$, and $\bar{V}^{\prime \prime}(0)=-H^{3} / 4$. Then $\|U-\bar{V}\|_{[0, L]}<3 / 4 c^{2} H^{-3-n}$ and $L<\bar{x}_{\text {min }}+12 c H^{-5-n}$ where $c=6.2^{2} e^{6}$.

Proof. Let $V$ be the solution of (6) with the same initial data as $U$. From the second inequality of estimate (22) it follows that $\|U-V\|_{[0, L]}<5 c H^{-3-n}$ for all $x \in[0, L]$. Therefore $V_{\text {min }}<5 c H^{-3-n}$. Property (P1) now implies that $10\left(\gamma+H^{3} / 4\right) / H^{2}<$ $5 c H^{-3-n}$ and hence $\gamma<-H^{3} / 4+c / 2 H^{-1-n}<-H^{3} / 4+3 H$. Lemma 12 now implies that $\|V-\bar{V}\|_{[0, L]}<20 e^{6} \mathrm{cH}^{-3-n}$. The triangle inequality then implies $\| U-$ $\bar{V} \|_{[0, L]}<3 / 4 c^{2} H^{-3-n}$.

Recall that $V_{\text {min }}=V\left(x_{\text {min }}\right)$ and by property (P3) $x_{\text {min }}<\bar{x}_{\text {min }}$. Further, Lemma 7 implies that $\bar{x}_{\text {min }}<L$. Arguing as in the final paragraph of the proof of Lemma 10, one can replace $X$ with $L$ and show that $V<3$ on $\left[x_{\text {min }}, L\right]$ hence $V^{\prime \prime}>H^{3} / 13$ on $\left[x_{\min }, L\right]$. Therefore $0=U^{\prime}(L)>V^{\prime}(L)-5 c / 5.9 H^{-2-n}$. Using that $V^{\prime}(L)>$ $H^{3} / 13\left(L-x_{\min }\right)$ we conclude $L-\bar{x}_{\min }<L-x_{\min }<12 c H^{-5-n}$, as desired.

Now we can conclude that the shape of the profiles $U$ is approaching the stationary solution as $H \rightarrow \infty$. Let $U_{s h}(z):=\frac{1}{H} U(L z)$ and $\bar{V}_{s h}:=\frac{1}{H} \bar{V}\left(\bar{x}_{\text {min }} z\right)$ for $z \in[0,1]$.

Corollary 14. Let $H>100$. Then $\left\|U_{s h}-\bar{V}_{s h}\right\|_{C^{2}[0,1]}<c^{2} H^{-4-n}$ where $c$ is as in Lemma 13.

In [25], Witelski et al. presented evidence for such a result. They considered source-type and blow-up selfsimilar solutions with droplet profiles, parametrizing them by the length of the support. Via asymptotics, they found that as the length tends to zero, the profiles of both the source-type and blow-up selfsimilar solutions are, to the first order, that of a droplet steady state. Also, they presented numerical evidence that as the length tends to zero, their masses tend to $M_{c}$. As the length of the selfsimilar solution tends to zero, the height tends to infinity and so their results are directly related to the above corollary. 
Proof. For $z \in[0,1]$

$$
\begin{aligned}
& \left|U_{s h}^{\prime \prime}(z)-\bar{V}_{s h}^{\prime \prime}(z)\right|=\left|\frac{L^{2}}{H} U^{\prime \prime}(L z)-\frac{\bar{x}_{\min }^{2}}{H} \bar{V}^{\prime \prime}\left(\bar{x}_{\min } z\right)\right| \\
& \leq\left|\frac{L^{2}}{H}\left(U^{\prime \prime}-\bar{V}^{\prime \prime}\right)(L z)\right|+\left|\frac{L^{2}-\bar{x}_{\min }^{2}}{H} \bar{V}^{\prime \prime}(L z)\right|+\left|\frac{\bar{x}_{\min }^{2}}{H}\left(\bar{V}^{\prime \prime}(L z)-\bar{V}^{\prime \prime}\left(\bar{x}_{\min } z\right)\right)\right| \\
& <\frac{1}{H}\|U-\bar{V}\|_{[0, L]}+12 c H^{-5-n} \frac{13}{H} \frac{1}{H} \frac{H^{3}}{4}+\frac{\bar{x}_{\min }^{2}}{H}\left(L-\bar{x}_{m i n}\right) \bar{V}^{2}(\xi)\left|\bar{V}^{\prime}(\xi)\right| \\
& <\frac{3 c^{2}}{4} H^{-4-n}+40 c H^{-4-n}+\left(\frac{6}{H}\right)^{2} \frac{1}{H} 12 c H^{-5-n} H^{2} \frac{H^{2}}{3} \\
& <c^{2} H^{-4-n} .
\end{aligned}
$$

In the above, $\xi \in\left(\bar{x}_{\text {min }}, L\right)$ is from an application of the mean value theorem. Using that $U_{s h}(0)=\bar{V}_{s h}(0)$ and $U_{s h}^{\prime}(0)=\bar{V}_{s h}^{\prime}(0)$ the claim now follows.

In some sense, if one views the mass as a bifurcation parameter then as the mass tends to $M_{c}$, the blow-up selfsimilar solution loses its timescale and so its tending to a droplet steady state is not surprising. There are other results in the literature that are reminiscent of this. For example, in [11] Bowen and King consider the thin-film equation $u_{t}=-\left(u^{n} u_{x x x}\right)_{x}$ on the interval $[-1,1]$ with boundary conditions that allow the solution to drain. Via asymptotics, one finds that there can be no draining for $n \geq 2$ and the authors use asymptotics and numerics to demonstrate that as $n \uparrow 2$ the draining selfsimilar solutions are, to first order, that of a droplet steady state. Another example is found in Bernoff and Witelski's study [7] of source-type selfsimilar solutions for $u_{t}=-\left(u^{n} u_{x x x}\right)_{x}$. In this case, the critical exponent is $n=3$. Using asymptotics and numerics, they demonstrate that as $n \rightarrow 3$ ] the profiles of the source-type self similar solutions are, to first order, that of a droplet steady state.

4.2. The $n \in(1,3 / 2)$ Case. For $n>1, U^{1-n}$ blows up as $U$ approaches zero and so estimating the difference between $U$ and $V$ is more delicate than in the $0<n \leq 1$ case of Lemma 10. We do the estimates in two steps. In the first step $U(x)$ assumed to be bounded below (i.e. $x$ is not near the contact line), and hence the $U^{1-n}(x)$ term does not have a significant effect. In the second step, we consider those $x$ that are near the contact line. Here, the difference between $U$ and $V$ (in $C^{2}$ ) can grow arbitrarily large if $U$ were to approach zero sufficiently slowly. But we have no a priori information on how fast may $U$ be approaching zero. In fact, our aim is to get this information by comparing $U$ to $V$. This seemingly circular situation is resolved by Lemma 18 . This lemma allows us to extend the bounds on the $U-V$ from $\gamma=-H^{3} / 4+H$ (for which $U$ can be analyzed using only the first step) to an appropriate subset of $\left[-H^{3} / 4,-H^{3} / 4+H\right] \cap S^{+}(H)$.

Lemma 15. Let $1<n<3 / 2$. Assume $U(0)=V(0)=H, U^{\prime}(0)=V^{\prime}(0)=0$, and $U^{\prime \prime}(0)=V^{\prime \prime}(0)=\gamma \in\left[-H^{3} / 4,-H^{3} / 4+H\right]$. Let $U$ and $V$ be the resulting solutions of equation (12) and equation (6) respectively. If $\gamma>-H^{3} / 4$ then $V$ is defined on $\mathbb{R}$. 
If $\gamma=-H^{3} / 4$ then $V$ is taken to be extended to $\mathbb{R}$ via formula (10). Define

$$
X_{\text {cut }}:=\sup \left\{x>0 \mid U^{\prime}<0 \text { and } U>\frac{1}{2 H} \text { on }(0, x)\right\} \text {. }
$$

If $H \geq 40$ then $5 / H<X_{\text {cut }}<6 / H$ and for all $x \in\left[0, X_{\text {cut }}\right]$

$$
|U(x)-V(x)|<\frac{9}{H},\left|U^{\prime}(x)-V^{\prime}(x)\right|<3,\left|U^{\prime \prime}(x)-V^{\prime \prime}(x)\right|<\frac{H}{2} .
$$



Figure 3: Phase diagram relevant to the proof of Lemma 15. Here we plot $\left(-U^{\prime}, U\right)$ and $\left(-V^{\prime}, V\right)$, parameterized by $x$, where $U$ and $V$ are solutions of equations (12) and (6) respectively. $U(0)=V(0)=H$, $U^{\prime}(0)=V^{\prime}(0)=0$ and $V^{\prime \prime}(0)=U^{\prime \prime}(0) \in S^{+} \cap\left[-H^{3} / 4,-H^{3} / 4+H\right]$. The bounds in Lemma 15 hold for $x \leq X_{\text {cut }}$ where $X_{\text {cut }}$ is determined by $U$. In the figure, $\alpha>X_{\text {cut }}$ is the first positive $x$ at which $U^{\prime}(x)=0$. Lemma 17 and Lemma 18 are concerned with $x \in\left(X_{c u t}, \alpha\right]$.

Proof. Let $x_{1}:=\min \left\{6 / H, X_{\text {cut }}\right\}$. We will prove that $x_{1}<6 / H$ and hence $X_{\text {cut }}<$ $6 / H$. Using the Grönwall inequality (18) combined with (21),

$$
g(x) \leq e^{6} \int_{0}^{x} \frac{s}{n+4} U(s)^{1-n} d s \quad \forall x \in\left(0, x_{1}\right)
$$

Therefore since $H \geq 40$,

$$
g\left(x_{1}\right) \leq e^{6} \frac{1}{n+4}\left(\frac{1}{2 H}\right)^{1-n} \frac{1}{2}\left(\frac{6}{H}\right)^{2}<\left[e^{6} \frac{1}{1+4} 2^{n-2} 40^{n-4} 36\right] H<\frac{1}{2} H .
$$

Because $g$ is nondecreasing, $\left|U^{\prime \prime}(x)-V^{\prime \prime}(x)\right|<H / 2$ for all $x \in\left[0, x_{1}\right]$. Integrating and using $x<6 / H$ then yields

$$
\left|U^{\prime}(x)-V^{\prime}(x)\right|<3 \quad \text { and } \quad|U(x)-V(x)|<\frac{9}{H} \quad \forall x \in\left[0, x_{1}\right] .
$$


We now prove that $x_{1}<6 / H$, and hence the above bounds hold on $\left[0, X_{c u t}\right]$, as desired. Assume not; assume $x_{1}=6 / H$. Note that $U^{\prime} \leq 0$ on $\left[0, x_{1}\right]$. Let $x_{2}$ be the lesser of $6 / H$ and the smallest $x>x_{\min }$ for which $V(x)=1$. If $V\left(x_{2}\right)=1$ then $V^{\prime}\left(x_{2}\right)=-p(1)>3$. Therefore $\left|V^{\prime}\left(x_{2}\right)-U^{\prime}\left(x_{2}\right)\right|>3$ which contradicts the estimate (25). Therefore we can assume that $V(x)<1$ on $\left(x_{\min }, 6 / H\right)$. It follows from equation (7) that $V^{\prime \prime}(x)>-H^{3} / 4+H^{3} / 3-1 / 3>H^{3} / 13$ on $\left(x_{\min }, 6 / H\right)$. Since $6 / H-x_{\min }>0.05 / H$ it follows that $V^{\prime}(6 / H)>0.003 H^{2}>3$, which leads to contradiction as before. Therefore $x_{1}<6 / H$, as desired. A similar argument (using property (P3)) shows that $X_{\text {cut }}>5 / H$.

We now show that $\gamma=-H^{3} / 4+H$ is in $S^{+}(H)$. Further, we show that in Figure 3 , the resulting curve $\left(-U^{\prime}, U\right)$ crosses the vertical axis with a fairly large "speed":

Lemma 16. Let $1<n<3 / 2$. Assume $U(0)=H$, $U^{\prime}(0)=0$, and $U^{\prime \prime}(0)=\gamma=$ $-H^{3} / 4+H$. Let $U$ be the resulting solution of equation (12). Let $S^{+}(H)$ and $S^{-}(H)$ be as defined in (24) and $\alpha(H, \gamma)$ be as defined in (23). If $H \geq 40$ then $\gamma=-H^{3} / 4+$ $H \in S^{+}(H) \backslash S^{-}(H)$. Furthermore $U^{\prime \prime}(\alpha(H, \gamma))>H^{3} / 23$.

Proof. Let $X:=X(H, \gamma), \alpha:=\alpha(H, \gamma)$ and $\beta:=\beta(H, \gamma)$. Recall from Lemma 15, $X_{\text {cut }}=\sup \left\{x>0 \mid U^{\prime}<0\right.$ and $U>\frac{1}{2 H}$ on $\left.(0, x)\right\}<6 / H$. Hence $X_{\text {cut }}=\alpha$, or $U\left(X_{\text {cut }}\right)=1 /(2 H)$. We claim that $X_{\text {cut }}=\alpha$. Assume not; then $U\left(X_{\text {cut }}\right)=$ $1 /(2 H)$. Combining this with the bound (25) yields $V\left(X_{c u t}\right)<10 / H$. This is impossible because Property (P1) in $\S 2$ implies that the smallest value $V$ achieves satisfies $V_{\min }>10 / H$. Therefore $X_{c u t}=\alpha$. By definition, $X_{c u t}<\beta$ and so $\alpha<\beta$ and thus $\gamma \in S^{+}(H) \backslash S^{-}(H)$.

The bound (25) then implies that $\left|V^{\prime}(\alpha)\right|<3$. By the formula (9) for $p(V)$ it then follows that $V(\alpha)<1$ and equation (7) then implies that $V^{\prime \prime}(\alpha) \geq H^{3} / 12-1 / 3>$ $H^{3} / 13$. Therefore bound (25) implies that $U^{\prime \prime}(\alpha)>V^{\prime \prime}(\alpha)-H / 2>H^{3} / 23$, as desired.

We now prove that if $\gamma$ is such that the resulting solution $U$ comes close to touching down in the first lap (i.e., $U(x)<1 /(2 H)$ for some $x<\alpha$ ) then $U^{\prime \prime \prime}$ can be controlled. This will be useful in arguing that an inflection point, like that of $U_{2}$ in Figure 2, cannot exist for $H$ sufficiently large.

Lemma 17. Let $1<n<3 / 2$. Assume $U(0)=H$, $U^{\prime}(0)=0$, and $U^{\prime \prime}(0)=\gamma \in$ $\left[-H^{3} / 4,-H^{3} / 4+H\right]$. Let $U$ be the resulting solution of equation (12). Let $X_{\text {cut }}$ be as defined in Lemma 15:

$$
X_{c u t}=\sup \left\{x>0 \mid U^{\prime}<0 \text { and } U>\frac{1}{2 H} \text { on }(0, x)\right\} .
$$

Assume $H \geq 40$. Let $\alpha(H, \gamma)$ be as defined in (23) and $S^{+}(H)$ be as defined in (24). If $\gamma \in S^{+}(H)$ and $X_{\text {cut }}<\alpha(H, \gamma)$ then for all $x \in\left(X_{\text {cut }}, \alpha(H, \gamma)\right), U^{\prime \prime \prime}(x)<0$ and $U^{\prime \prime}(x)>0$.

Proof. Let $\alpha:=\alpha(H, \gamma)$. By assumption, $X_{\text {cut }}<\alpha$ and so $U\left(X_{\text {cut }}\right)=1 /(2 H)$. We now find bounds for $U^{\prime}\left(X_{c u t}\right), U^{\prime \prime}\left(X_{\text {cut }}\right)$, and $U^{\prime \prime \prime}\left(X_{\text {cut }}\right)$. Using bound (25), $V\left(X_{\text {cut }}\right)<\frac{1}{2 H}+\frac{9}{H}$. Therefore equation (9) implies $\left|V^{\prime}\left(X_{\text {cut }}\right)\right|=-p\left(V\left(X_{\text {cut }}\right)\right)<$ 
$\sqrt{H(9.5 H+1) / 6}<\sqrt{5 / 3} H$. Estimate (25) then yields $\left|U^{\prime}\left(X_{c u t}\right)\right|<\sqrt{5 / 3} H+$ $3<2 H$. Also note that equation (7) implies $U^{\prime \prime}\left(X_{\text {cut }}\right) \geq V^{\prime \prime}\left(X_{\text {cut }}\right)-H / 2 \geq$ $-H^{3} / 4+H^{3} / 3-(10 / H)^{3} / 3-H / 2>H^{3} / 13$. Using equation (12) and the lower bound $5 / H<X_{\text {cut }}$ of Lemma 15 , one finds $U^{\prime \prime \prime}\left(X_{\text {cut }}\right)<0$.

Assume $U^{\prime \prime \prime} \nless 0$ on $\left(X_{c u t}, \alpha\right)$. Then there exists a smallest $x_{3} \in\left(X_{c u t}, \alpha\right)$ such that $U^{\prime \prime \prime}\left(x_{3}\right)=0$. From the equation (12), and since $U\left(x_{3}\right)<1 /(2 H)$, it follows that

$$
0=U^{\prime \prime \prime}\left(x_{3}\right)=-\frac{x_{3}}{n+4} U\left(x_{3}\right)^{1-n}-U\left(x_{3}\right)^{2} U^{\prime}\left(x_{3}\right)<-\frac{10}{11 H}-\frac{1}{4 H^{2}} U^{\prime}\left(x_{3}\right)
$$

and hence $U^{\prime}\left(x_{3}\right)<-\frac{7}{2} H<U^{\prime}\left(X_{c u t}\right)$. Therefore $U^{\prime \prime}(x)<0$ for some $x \in$ $\left(X_{c u t}, x_{3}\right)$. Since $U^{\prime \prime}\left(X_{c u t}\right)>0$ and $U^{\prime \prime \prime}<0$ on $\left(X_{c u t}, x_{3}\right)$ there exists a unique $x_{2} \in\left(X_{\text {cut }}, x_{3}\right)$ such that $U^{\prime \prime}\left(x_{2}\right)=0$. Furthermore $U^{\prime \prime}\left(x_{3}\right)<0$.

Since $U^{\prime \prime}(\alpha) \geq 0$ there exists a smallest $x_{4}>x_{3}$ such that $U^{\prime \prime}\left(x_{4}\right)=0$. Integrating the equation (12) yields

$$
U^{\prime \prime}=\gamma+\frac{H^{3}}{3}-\frac{U^{3}}{3}-\frac{1}{n+4} \int_{0}^{x} s U(s)^{1-n} d s .
$$

Since $U^{\prime \prime}\left(x_{2}\right)=U^{\prime \prime}\left(x_{4}\right)$

$$
\left(\frac{1}{2 H}\right)^{3}>U\left(x_{2}\right)^{3}-U\left(x_{4}\right)^{3}=\frac{3}{n+4} \int_{x_{2}}^{x_{4}} s U(s)^{1-n} d s>\frac{x_{4}^{2}-x_{2}^{2}}{4}>\frac{2}{H}\left(x_{4}-x_{2}\right) .
$$

Hence $x_{4}-x_{2}<H^{-2}$.

Since $U^{\prime \prime}>0$ on $\left[X_{c u t}, x_{2}\right)$, it follows that $U^{\prime}\left(x_{2}\right)>U^{\prime}\left(X_{\text {cut }}\right)>-2 H$. Similarly, $U^{\prime \prime}<0$ on $\left(x_{3}, x_{4}\right)$ and so $U^{\prime}\left(x_{4}\right)<U^{\prime}\left(x_{3}\right)<-7 / 2 H$. Therefore $U^{\prime}\left(x_{4}\right)-U^{\prime}\left(x_{2}\right)<-H / 2$ and the mean value theorem implies there exists $x_{5} \in$ $\left(x_{2}, x_{4}\right)$ such that $U^{\prime \prime}\left(x_{5}\right)<-H^{3} / 2$. Therefore

$$
\begin{aligned}
0=U^{\prime \prime}\left(x_{4}\right) & =U^{\prime \prime}\left(x_{5}\right)+\frac{U\left(x_{5}\right)^{3}-U\left(x_{4}\right)^{3}}{3}-\frac{1}{n+4} \int_{x_{5}}^{x_{4}} s U(s)^{1-n} d s \\
& <-\frac{H^{3}}{2}+\frac{1}{3}\left(\frac{1}{2 H}\right)^{3}<0 .
\end{aligned}
$$

This contradiction proves $U^{\prime \prime \prime}<0$ on $\left(X_{c u t}, \alpha\right)$, as desired.

Finally, since $U^{\prime \prime}(\alpha) \geq 0$, the negativity of $U^{\prime \prime \prime}$ implies that $U^{\prime \prime}(x)>0$ for $x \in$ $\left(X_{c u t}, \alpha\right)$.

Lemma 18. Let $1<n<3 / 2$. Assume $H \geq \max \left\{40,3(3-2 n)^{-2 / 11}\right\}$ and $\gamma \in$ $S^{+}(H) \cap\left[-H^{3} / 4,-H^{3} / 4+H\right]$ where $S^{+}(H)$ is as defined in (24). $U$ is the solution of equation (12) with $U(0)=H, U^{\prime}(0)=0$, and $U^{\prime \prime}(0)=\gamma$. Let $\alpha(H, \gamma)$ be as defined in (23). If $U^{\prime \prime}(\alpha(H, \gamma))>H^{3} / 24$ then $U^{\prime \prime}(\alpha(H, \gamma))>H^{3} / 23$.

Proof. Let $\alpha:=\alpha(H, \gamma)$. Recall that $X_{\text {cut }}:=\sup \left\{x>0 \mid U^{\prime}<0\right.$ and $U>$ $1 /(2 H)$ on $(0, x)\}<6 / H$ by Lemma 15. If $X_{c u t}=\alpha$ then arguing as in the proof of Lemma 16 yields $U^{\prime \prime}(\alpha)>H^{3} / 23$. 
Now consider the case $X_{\text {cut }}<\alpha$. Then $U\left(X_{\text {cut }}\right)=1 /(2 H)$. Since $U^{\prime \prime}$ is decreasing on $\left(X_{\text {cut }}, \alpha\right)$ (by Lemma 17) and $U^{\prime \prime}(\alpha)>H^{3} / 24$, it follows that $U^{\prime \prime}>H^{3} / 24$ on $\left(X_{c u t}, \alpha\right)$. Hence

$$
U(x) \geq U(\alpha)+\frac{H^{3}}{48}(x-\alpha)^{2} \quad \forall x \in\left(X_{\text {cut }}, \alpha\right) .
$$

Specifically $1 /(2 H) \geq H^{3} / 48\left(\alpha-X_{\text {cut }}\right)^{2}$, implying $\Delta x:=\alpha-X_{\text {cut }}<5 / H^{2}$. This implies

$$
\alpha<6 / H+5 / H^{2}<6.2 / H
$$

Let $V$ be the solution of equation (6) with same initial data as $U$. Using bound (25), $V\left(X_{\text {cut }}\right)<10 / H$. Recalling that $\left|V^{\prime}\right|<H^{2} / 3$ by Property (P2) in $\S 2, V(x)<$ $10 / H+\left(H^{2} / 3\right) \cdot\left(5 / H^{2}\right)<2$ for all $x \in\left(X_{c u t}, \alpha\right)$. We will now use the Grönwall inequality (19). To do this we first bound

$$
\int_{X_{\text {cut }}}^{\alpha} V(s)\left|V^{\prime}(s)\right| s^{2}+V^{2}(s) s d s<2 \frac{H^{2}}{3} \frac{6.1^{2}}{H^{2}} \frac{5}{H^{2}}+4 \frac{6.1}{H} \frac{5}{H^{2}}<0.09
$$

To apply the Grönwall inequality (18), we denote $\psi(s):=V(s)\left|V^{\prime}(s)\right| s^{2}+V^{2}(s)$ and $\phi(s):=\frac{1}{n+4} s U^{1-n}(s)$. Combined with the inequality above, the inequality (21) implies that $\int_{0}^{\alpha} \psi(s) d s<e^{6}$. Using the inequality (26) it then follows

$$
\begin{aligned}
g(\alpha) & \leq \int_{0}^{\alpha} \phi(s) e^{\int_{s}^{\alpha} \psi(z) d z} d s \\
& \leq e^{\int_{0}^{\alpha} \psi(s) d s} \int_{0}^{X_{c u t}} \phi(s) d s+e^{\int_{X_{c u t}}^{\alpha} \psi(s) d s} \int_{X_{\text {cut }}}^{\alpha} \phi(s) d s \\
& <\frac{H}{2}+\frac{e^{0.09}}{n+4} \int_{X_{\text {cut }}}^{\alpha} s U(s)^{1-n} d s \\
& <H+\frac{1.1}{5} \int_{X_{\text {cut }}}^{\alpha} s\left(\frac{H^{3}}{48}(s-\alpha)^{2}\right)^{1-n} d s \\
& <H+\frac{1.1}{5} \frac{6.1}{H} \frac{H^{3(1-n)}}{48^{1-n}} \frac{\Delta x^{3-2 n}}{3-2 n} \\
& <H+10 \frac{H^{n-4}}{3-2 n}
\end{aligned}
$$

Therefore $\left|U^{\prime \prime}(\alpha)-V^{\prime \prime}(\alpha)\right|<H+10 H^{n-4} /(3-2 n)$. Because $400 /(3-2 n)<$ $H^{11 / 2}<H^{7-n}$, it follows that $U^{\prime \prime}(\alpha)>V^{\prime \prime}(\alpha)-H^{3} / 40-H>H^{3} / 23$, as desired. In the last step, we used equation (7) to find $V^{\prime \prime}(\alpha)>H^{3} / 12-2^{3} / 3>H^{3} / 13$.

We can now prove the existence result:

Theorem 19. Assume $1<n<3 / 2$. Given $H \geq \max \left\{40,3(3-2 n)^{-2 / 11}\right\}$, there exists $\gamma \in\left[-H^{3} / 4,-H^{3} / 4+H\right]$ such that the solution of (12) with $U(0)=H$, $U^{\prime}(0)=0$, and $U^{\prime \prime}(0)=\gamma$ has compact support $[-L, L], U^{\prime}( \pm L)=0$, and $U$ is decreasing on $[0, L]$. 
Proof. Let $S^{+}:=S^{+}(H)$ and $S^{-}:=S^{-}(H)$ be as defined in (24). By Lemma 16, $-H^{3} / 4+H \in S^{+}(H)$. Let

$$
\gamma_{1}:=\inf \left\{\gamma \mid \tilde{\gamma} \in\left[\gamma,-H^{3} / 4+H\right] \Longrightarrow \tilde{\gamma} \in S^{+} \text {and } U(\alpha(H, \tilde{\gamma}))>0\right\}
$$

where $U$ is the solution of (12) with initial data $U(0)=H, U^{\prime}(0)=0$, and $U^{\prime \prime}(0)=\tilde{\gamma}$ and $\alpha(H, \tilde{\gamma})$ is as defined in (23). Note that $-H^{3} / 4 \leq \gamma_{1}$ since $-H^{3} / 4 \in S^{-} \backslash S^{+}$ by Corollary 6. We will show that $U$, the solution of (12) with $U^{\prime \prime}(0)=\gamma_{1}$, touches down with zero contact angle and is decreasing up to the touch down. More precisely we show that $U\left(\alpha\left(H, \gamma_{1}\right)\right)=0$.

Let $A:=\left\{\gamma \in S^{+} \mid U^{\prime \prime}(\alpha(H, \gamma)) \geq H^{3} / 23\right\}$. By Lemma 16, $-H^{3} / 4+H \in A$ and hence $A$ is nonempty.

We claim that $A$ is closed in $\left[\gamma_{1},-H^{3} / 4+H\right]$. Let $\left\{\gamma_{m}\right\} \subset A \cap\left[\gamma_{1},-H^{3} / 4+H\right]$ be a convergent sequence with limit $\gamma$. We will prove that $\gamma \in A$. Let $U_{m}$ and $U$ be the corresponding solutions of (12). Arguing as for (27) one finds that $\alpha(H, \gamma)$ is uniformly bounded for $\gamma \in A$ and so the sequence $\alpha_{m}:=\alpha\left(H, \gamma_{m}\right)$ has a subsequence converging to some $\hat{\alpha}$. To keep the notation simple we assume that the whole sequence converges. From (13) it follows that $H^{3} / 3>\left|U_{m}^{\prime \prime}\right|$ on $\left[0, \alpha_{m}\right]$. Therefore by the Arzela-Ascoli lemma there exists a subsequence, which we again assume to be the whole sequence $U_{m}$, such that $U_{m} \rightarrow U$ and $U_{m}^{\prime} \rightarrow U^{\prime}$ uniformly on compact subsets of $[0, \hat{\alpha})$ and pointwise on $[0, \hat{\alpha})$. By continuous dependence of solutions of (12) on initial data, $U$ is a solution of the equation with initial data $U(0)=H, U^{\prime}(0)=0$, and $U^{\prime \prime}(0)=\gamma$ on the interval where $U$ is positive.

We now show that $U$ is positive on $[0, \hat{\alpha})$. If not, then there exists the smallest positive $x<\hat{\alpha}$, call it $x_{0}$, for which $U$ is zero. For $m$ sufficiently large, $x_{0}<\alpha_{m}$. Since $U_{m}^{\prime \prime}\left(\alpha_{m}\right) \geq H^{3} / 23$ it follows from Lemma 17 that either $U_{m}\left(x_{0}\right) \geq 1 /(2 H)$ or $U_{m}\left(x_{0}\right) \geq H^{3} / 46\left(\alpha_{m}-x_{0}\right)^{2}$. Therefore $U\left(x_{0}\right)=\lim _{m \rightarrow \infty} U_{m}\left(x_{0}\right)>0$, contradicting $U\left(x_{0}\right)=0$.

Thus $U$ is a solution of (12) on $[0, \hat{\alpha})$. By continuous dependence of solutions on initial data $U_{m}^{\prime \prime}$ converges to $U^{\prime \prime}$ pointwise on $[0, \hat{\alpha})$. Also $U^{\prime} \leq 0$ on $[0, \hat{\alpha})$ and so $U(\hat{\alpha}):=\lim _{x \rightarrow \hat{\alpha}^{-}} U(x)$ exists. We now argue that $\alpha:=\alpha(H, \gamma) \geq \hat{\alpha}$. Assume not. Since $U$ is decreasing on $[0, \hat{\alpha})$, one has $U^{\prime}(\alpha)=0$ and $U^{\prime \prime}(\alpha)=0$. There are two cases to consider: $U(\alpha) \geq 1 /(2 H)$ and $U(\alpha)<1 /(2 H)$. If $U(\alpha) \geq 1 /(2 H)$ then Lemma 15 holds on $[0, \alpha]$. Specifically, $\left|V^{\prime \prime}(\alpha)\right|<H / 2$, implying $H / 2<V(\alpha)<$ $2 H / 3$ by equation (7). Using the formula for $p(V)$ given after (P3), it follows that $\left|V^{\prime}(\alpha)\right| \geq H^{2} / 9$. But this is impossible because Lemma 15 and $U^{\prime}(\alpha)=0$ imply $\left|V^{\prime}(\alpha)\right| \leq 3$. If $U(\alpha)<1 /(2 H)$ then for $m$ sufficiently large $U_{m}(\alpha)<1 /(2 H)$ and $\alpha<\alpha_{m}$. Applying Lemma 17 to the interval $\left(\alpha, \alpha_{m}\right)$, it follows that $U_{m}^{\prime \prime}(\alpha)>H^{3} / 23$ hence $U^{\prime \prime}(\alpha) \geq H^{3} / 23$. This contradicts $U^{\prime \prime}(\alpha)=0$, proving $\alpha \geq \hat{\alpha}$.

If $U(\hat{\alpha})>0$ then $\hat{\alpha}<\beta(H, \gamma)$ and the solution $U$ can be continued slightly beyond $\hat{\alpha}$. Continuous dependence of solutions on initial data then implies that $U_{m}^{\prime}\left(\alpha_{m}\right)$ converges to $U^{\prime}(\hat{\alpha})$ and hence $U^{\prime}(\hat{\alpha})=0$. Therefore $\alpha=\hat{\alpha}$ and so $\gamma \in S^{+}$. Furthermore, $U_{m}^{\prime \prime}\left(\alpha_{m}\right)$ converges to $U^{\prime \prime}(\alpha)$ and therefore $U^{\prime \prime}(\alpha) \geq H^{3} / 23$, proving that $\gamma \in A$, as desired. 
We now consider the case $U(\hat{\alpha})=0$. Here, $\hat{\alpha}=\beta(H, \gamma)$. Since $U_{m}$ are decreasing on $\left[0, \alpha_{m}\right), U_{m}$ converges to $U$ on $[0, \hat{\alpha})$, and $U$ takes arbitrarily small values near $\hat{\alpha}$, we conclude that $\lim _{m \rightarrow \infty} U_{m}\left(\alpha_{m}\right)=0$. Since $U_{m}^{\prime \prime}$ is uniformly bounded on $\left[0, \alpha_{m}\right]$, the fact that $U_{m}^{\prime}\left(\alpha_{m}\right)=0$ implies that $U^{\prime}(x) \rightarrow 0$ as $x \rightarrow \hat{\alpha}$ and hence $U^{\prime}(\hat{\alpha})=0$. Thus $\alpha=\hat{\alpha}$ and so $\gamma \in S^{+}$. It remains to prove that $U^{\prime \prime}(\alpha) \geq H^{3} / 23$. Note that for $m$ large enough $U_{m}\left(\alpha_{m}\right)<1 /(4 H)$. Therefore, since $U_{m}^{\prime \prime}<H^{3} / 3$, it follows that $U_{m}<1 /(2 H)$ on $\left(\alpha_{m}-1 / H^{2}, \alpha_{m}\right)$. Lemma 17 then implies that $U_{m}^{\prime \prime}$ is decreasing and is greater than $H^{3} / 23$ on $\left(\alpha_{m}-1 / H^{2}, \alpha_{m}\right)$. Therefore $U^{\prime \prime}$ is decreasing and is greater than or equal to $H^{3} / 23$ on $\left(\alpha-1 / H^{2}, \alpha\right)$. Therefore $U^{\prime \prime}(\alpha)=\lim _{x \rightarrow \alpha^{-}} U^{\prime \prime}(x) \geq$ $H^{3} / 23$, proving that $\gamma \in A$, as desired.

We now prove that $A$ is open in $\left(\gamma_{1},-H^{3} / 4+H\right]$. Fix $\tilde{\gamma} \in A \cap\left(\gamma_{1},-H^{3} / 4+H\right]$. Since $U(\alpha(H, \tilde{\gamma}))>0$ and $U^{\prime \prime}(\alpha(H, \tilde{\gamma})) \geq H^{3} / 23$, continuous dependence on initial data implies there exists a neighborhood $O \subset\left(\gamma_{1},-H^{3} / 4+H\right]$ of $\tilde{\gamma}$ such that for all $\gamma \in O$, one has $U(\alpha(H, \gamma))>0$ and $U^{\prime \prime}(\alpha(H, \gamma))>H^{3} / 24$. By construction, $\left(\gamma_{1},-H^{3} / 4+H\right] \subset S^{+}(H)$ and so Lemma 18 implies $U^{\prime \prime}(\alpha(H, \gamma))>H^{3} / 23$ for all $\gamma \in O$. This proves $O \subset A$, as desired.

The set $A$ is closed in $\left[\gamma_{1},-H^{3} / 4+H\right]$ and so it is also closed in $\left(\gamma_{1},-H^{3} / 4+\right.$ $H]$. The interval $\left(\gamma_{1},-H^{3} / 4+H\right]$ is connected and so $\left(\gamma_{1}, H^{3} / 4+H\right] \subset A$. It then follows that $\left[\gamma_{1},-H^{3} / 4+H\right] \subset A$ (because $A$ is closed in $\left[\gamma_{1},-H^{3} / 4+H\right]$ ). Therefore, $\gamma_{1} \in A$ and so $-H^{3} / 4<\gamma_{1} \in S^{+}$and $U^{\prime \prime}\left(\alpha\left(H, \gamma_{1}\right)\right)>0$. We now argue that $U\left(\alpha\left(H, \gamma_{1}\right)\right)=0$. If $U\left(\alpha\left(H, \gamma_{1}\right)>0\right.$, there exists a neighborhood of $\gamma_{1}$ in $\left(-H^{3} / 4,-H^{3} / 4+H\right]$ that is contained in $S^{+}$(see Figure 2). Further, by continuous dependence on initial data one can choose the neighborhood such that $U(\alpha(H, \gamma))>0$ for all $\gamma$ in the neighborhood. This contradicts $\gamma_{1}$ 's definition as an infimum. Therefore $U\left(\alpha\left(H, \gamma_{1}\right)\right)=0$, as desired.

Let $H_{\min }(n)$ be the infimum of the set of $H$ 's for which there exist zero-contactangle solutions $U$ that are symmetric about $x=0$ and have compact support. Theorem 19 gives an upper bound on $H_{\min }(n)$ and Theorem 9 gives a lower bound:

$$
(3-2 n)^{-2 / 11} \leq H_{\min }(n) \leq \max \left\{40,3(3-2 n)^{-2 / 11}\right\} \quad \forall n \in(1,3 / 2) .
$$

That is, $H_{\min }(n) \sim(3-2 n)^{-2 / 11}$ as $n$ increases to $3 / 2$.

Lemma 20. Let $H>\max \left\{40,3(3-2 n)^{-2 / 11}\right\}$ and $\gamma \in\left[\gamma_{1}, \frac{-H^{3}}{4}+H\right]$ where $\gamma_{1}$ is as in the proof of Theorem 19. Let $U$ be the solution of (12) with $U(0)=H, U^{\prime}(0)=0$, and $U^{\prime \prime}(0)=\gamma$ and $V$ the solution of (6) with the same initial data. Then $\| U-$ $V \|_{[0, \alpha(H, \gamma)]}<c H^{n-5}$ where $c=(6.1)^{2}\left(12 e^{6}+10(3-2 n)^{-9 / 11}\right)$.

Proof. We start by applying the arguments of the proof of Lemma 15. Recall that $X_{\text {cut }}:=\max \left\{x \mid x \leq \alpha(H, \gamma)\right.$ and $\left.U(x) \geq \frac{1}{2 H}\right\}$. By Lemma 15, $X_{\text {cut }}<6 / H$. From inequality (26) follows that for $x \in\left[0, X_{\text {cut }}\right]$

$$
g(x) \leq \frac{6^{2} \sqrt{2}}{10} e^{6} H^{n-3}
$$

where $g(x)$ is as before the $\max \left\{\left|U^{\prime \prime}(s)-V^{\prime \prime}(s)\right| \mid s \in[0, x]\right\}$. 
If $\alpha>X_{\text {cut }}$ we also apply the techniques of the proof of Lemma 18. Note that since $\gamma>\gamma_{1}$ the conditions of Lemma 18 are satisfied. From (28) now follows that $g(x) \leq 2 g\left(X_{\text {cut }}\right)+10 H^{n-4} /(3-2 n)$ for $x \in\left[X_{c u t}, \alpha\right]$. Combining the two inequalities implies that

$$
g \leq\left(12 e^{6}+10(3-2 n)^{-9 / 11}\right) H^{n-3}
$$

on $\left[X_{c u t}, \alpha\right]$, which after integrating twice implies the claim.

Corollary 21. Let $H>\max \left\{100,3(3-2 n)^{-2 / 11}\right\}$ and let $U$ be the solution of (12) with $U(0)=H, U^{\prime}(0)=0$ and $U^{\prime \prime}(0)=\gamma_{1}$. Let $\bar{V}$ be the solution of (6) with $\bar{V}(0)=H, \bar{V}^{\prime}(0)=0$, and $\bar{V}^{\prime \prime}(0)=-H^{3} / 4$. Then $\|U-\bar{V}\|_{[0, L]}<5 c e^{6} H^{n-5}$ where $c$ was defined in the lemma above and $L$ is the point where $U$ touches down. Furthermore $L<\bar{x}_{\min }+3 c H^{n-7}$.

Proof. Recall that by Theorem 19, $U$ touches down with zero contact angle and that by Lemma $18, L<6.1 / H$. Let $V$ be the solution of (6) with the same initial data as $U$. From the lemma above it follows that $\|U-V\|_{[0, L]}<c H^{n-5}$ for all $x \in[0, L]$. Therefore $V_{\text {min }}<c H^{n-5}$. Property (P1) now implies that $10\left(\gamma+H^{3} / 4\right) / H^{2}<$ $c H^{n-5}$ and hence $\gamma<-H^{3} / 4+\frac{c}{10} H^{n-3}<-H^{3} / 4+H$. Lemma 12 now implies that $\|V-\bar{V}\|_{[0, L]}<4 c e^{6} H^{n-5}$. Combining the inequalities now implies the first claim of the lemma. Arguing as in the proof of Lemma 13 one obtains that $L<x_{\min }+$ $3 c H^{n-7}$.

The following corollary shows that as $H$ grows the shape of the blowup solution approaches, in $C^{2}$, the shape of a steady state. The claim follows by repeating the argument of Corollary 14.

Corollary 22. Let $H$ and $U$ be as above. Then $\left\|U_{s h}-\bar{V}_{s h}\right\|_{C^{2}[0,1]}<6 c e^{6} H^{n-6}$ and $\left\|U_{s h}-\bar{V}_{s h}\right\|_{L^{\infty}[0,1]}<1 / H$.

\section{EXISTENCE OF MULTI-BUMP ZERO-CONTACT-ANGLE SOLUTIONS}

A selfsimilar solution of the thin-film equation is said to have $k$ bumps if it has exactly $k$ local maxima. For solution that is symmetric about $x=0$ this corresponds to the solution of equation (12) having $k$ positive local extrema for $x \geq 0$. In this section we show that for any $k$ there exists a compactly supported symmetric solution of (12) with zero contact angles and $k$ bumps. This is done via a shooting method using estimates we established in $\S 2$ and $\S 4$. In the following, we outline the proof when $n \in(1,3 / 2)$. The proof for $n \in(0,1]$ is analogous but simpler.

We first consider the case $k$ is odd. Let $U$ be a solution of (12) with $U(0)=$ $H, U^{\prime}(0)=0$ and $U^{\prime \prime}(0)=\gamma<0$. Consider all points $x \geq 0$ such that $U^{\prime}(x)=0$ and $U(x)$ is a local extremum. This excludes inflection points and it excludes the contact line if the contact angle is nonzero. Let $\alpha_{1}=0<\alpha_{2}<\alpha_{3}, \ldots$ be all such points. If $\alpha_{k+1}$ exists but $\alpha_{k+2}$ does not exist, then $U$ is symmetric, has $k$ bumps and zero contact angles. In $\S 4$, we proved that if $H$ is sufficiently large, then one can find $\gamma$ such that $\alpha_{2}(=\alpha(H, \gamma))$ exists but $\alpha_{3}$ does not. Let $V$ be the solution of (6) with the 
same initial data as $U$. Note that $V^{\prime \prime}$ is large where $V^{\prime}=0$. One of the key steps in $\S 4$ was to prove that if $H$ is large enough, then $U$ is sufficiently close to $V$ to ensure $U$ has no inflection points where $U^{\prime}=0$, in its first lap. This allowed us to carry out the topological arguments needed for the shooting argument.

To prove the existence of multi-bump solutions with zero contact angles, we will argue similarly. Consider $V$ on $\left[0,(k+1) x_{\min }\right]$, where $2 x_{\min }$ is the period of $V$. Similar methods to those that yielded the bound on $\|U-V\|_{\left[0, \alpha_{2}\right]}$ in Lemma 20 can be used to bound $\|U-V\|_{\left[0, \alpha_{k+1}\right]}$ if $H$ is large enough. (The larger $k$ is, the larger $H$ must be taken.) In [25], Witelski, Bernoff, and Bertozzi computationally observed this closeness between $U$ and $V$ for multi-bump solutions. This closeness will then imply that $U$ has no inflection points where $U^{\prime}=0$ in its first $k / 2$ laps and, in particular (for appropriate set of $\gamma), U^{\prime \prime}\left(\alpha_{k+1}\right)>\varepsilon>0$. This nondegeneracy condition will then enable us to use topological arguments.

5.1. Bounding $U-V$ for one lap. The estimates over a number of laps of $U$ starting from 0 will be deduced from an estimate on a single lap of $U$ that starts at $x_{0} \geq 0$. For this reason, in the following we introduce $x_{0}$ as the location of the initial data. If $U\left(x_{0}\right)=H, U^{\prime}\left(x_{0}\right)=0$, and $U^{\prime \prime}\left(x_{0}\right)=\gamma$ and $U$ is the resulting solution of (12), we define $\alpha_{1}\left(x_{0}\right)=x_{0}<\alpha_{2}\left(x_{0}\right)<\alpha_{3}\left(x_{0}\right)<\ldots$ as above. We define

$$
\begin{aligned}
& S_{k}^{+}\left(H, x_{0}\right)=\left\{\gamma \leq 0 \mid \alpha_{k+1}\left(x_{0}\right) \text { exists }\right\}, \\
& S_{k}^{-}\left(H, x_{0}\right)=\left\{\gamma \leq 0 \mid \alpha_{k+2}\left(x_{0}\right) \text { does not exist }\right\} .
\end{aligned}
$$

We first state a technical lemma concerning the steady states. Its proof is similar to the proof of Lemma 12 so we omit it.

Lemma 23. Let $V_{1}$ and $V_{2}$ be two solutions of (6) such that $V_{1}$ is nonnegative and has maximum $H$. For every $a>0$ and every $\varepsilon>0$ there exists $\delta>0$ such that if $\left|V_{1}(0)-V_{2}(0)\right|<\delta H^{-a-2},\left|V_{1}^{\prime}(0)-V_{2}^{\prime}(0)\right|<\delta H^{-a-1}$, and $\left|V_{1}^{\prime \prime}(0)-V_{2}^{\prime \prime}(0)\right|<$ $\delta H^{-a}$ then $\left\|V_{1}-V_{2}\right\|_{[0,13 / H]}<\varepsilon H^{-a-2}$. Although equation (6) is presented with the assumption that $\gamma<0$, these bounds hold regardless of the sign of $\gamma$.

Lemmas 16, 17, and 18 are stated for $U$ with initial data specified at $x_{0}=0$. One can easily check that if the initial data had been specified at $x_{0}>0$ the analogous lemmas would still hold although one would have to choose $H$ large enough (depending on $x_{0}$ ). These analogous lemmas are assumed in the following. In particular the analogue of Lemma 16 implies that for $H$ large enough $-H^{3} / 4+H \in S_{1}^{+}\left(H, x_{0}\right)$. This allows us to define

$$
\gamma_{1}\left(x_{0}\right):=\inf \left\{\gamma \mid \tilde{\gamma} \in\left[\gamma,-\frac{H^{3}}{4}+H\right] \Rightarrow \tilde{\gamma} \in S_{1}^{+}\left(H, x_{0}\right) \text { and } U\left(\alpha_{2}\left(H, \tilde{\gamma}, x_{0}\right)\right)>0\right\} \text {. }
$$

The following states that if $H$ is sufficiently large, $U$ and $V$ are close to each other for the first downstroke of $U:\left[x_{0}, \alpha_{2}\left(x_{0}\right)\right]$. Its proof is very similar to the proof of Lemma 20 and so we omit it.

Lemma 24. Let $1<n<3 / 2, C>0$, and $\varepsilon \in(0,1)$. Then there exists $H_{1}$ such that if $H \geq H_{1}, x_{0} \in[0, C / H], U\left(x_{0}\right)=V\left(x_{0}\right)=H, U^{\prime}\left(x_{0}\right)=V^{\prime}\left(x_{0}\right)=0$, 
$U^{\prime \prime}\left(x_{0}\right)=V^{\prime \prime}\left(x_{0}\right)=\gamma \in\left[\gamma_{1}\left(x_{0}\right),-H^{3} / 4+H\right]$ and $U$ and $V$ are the resulting solutions of (12) and (6) respectively then $\|U-V\|_{\left[x_{0}, \alpha_{2}\left(x_{0}\right)\right]}<\varepsilon H^{-7 / 2}$. Furthermore $x_{0}+5 / H<\alpha_{2}\left(x_{0}\right)<x_{0}+6.1 / H$.

We now prove that by taking $H$ larger still, they are close to each other for the lap of $U:\left[x_{0}, \alpha_{3}\left(x_{0}\right)\right]$.

Lemma 25. Let $1<n<3 / 2, C>0$, and $\varepsilon \in(0,1)$. Then there exists $\mathrm{H}_{2}$ such that if $H \geq H_{2}, x_{0} \in[0, C / H], U\left(x_{0}\right)=V\left(x_{0}\right)=H, U^{\prime}\left(x_{0}\right)=V^{\prime}\left(x_{0}\right)=0$, $U^{\prime \prime}\left(x_{0}\right)=\bar{V}^{\prime \prime}\left(x_{0}\right) \in\left(\gamma_{1}\left(x_{0}\right),-H^{3} / 4+H\right]$ and $U$ and $V$ are the resulting solutions of (12) and (6) respectively then $\|U-V\|_{\left[x_{0}, \alpha_{3}\left(x_{0}\right)\right]}<\varepsilon H^{-7 / 2}$. Furthermore, $\alpha_{2}\left(x_{0}\right)+$ $5 / H<\alpha_{3}\left(x_{0}\right)<\alpha_{2}\left(x_{0}\right)+6.1 / H$.

Proof. Let $\alpha_{2}=\alpha_{2}\left(x_{0}\right)$ and $\alpha_{3}=\alpha_{3}\left(x_{0}\right)$. Lemma 24 provides the closeness for the first downstroke of $U$. For the upstroke of $U$ we introduce $V_{2}$, the solution of (6) with $V_{2}\left(\alpha_{2}\right)=U\left(\alpha_{2}\right), V_{2}^{\prime}\left(\alpha_{2}\right)=U^{\prime}\left(\alpha_{2}\right)=0$, and $V_{2}^{\prime \prime}\left(\alpha_{2}\right)=U^{\prime \prime}\left(\alpha_{2}\right)$. By Lemma 23, we can assume that $\left\|V_{2}-V\right\|_{\left[\alpha_{2}, \alpha_{3}\right]}<\frac{\varepsilon}{10} H^{-7 / 2}$. And so to prove the first part of the Lemma, it suffices to show that $\left\|U-V_{2}\right\|_{\left[\alpha_{2}, \alpha_{3}\right]}<\frac{\varepsilon}{10} H^{-7 / 2}$.

Let $x_{\max }$ be the location of the first maximum of $V_{2}$ after $\alpha_{2}$. Arguing as in Lemma 5 yields that $V_{2}>U$ on $\left(\alpha_{2}, x_{\max }\right)$. Recall that $U<H$ on $\left(\alpha_{2}, \alpha_{3}\right)$ by Lemma 1 . Let $g(x):=\max \left\{\left|U^{\prime \prime}(s)-V_{2}^{\prime \prime}(s)\right| \mid s \in\left(\alpha_{2}, x\right)\right\}$. Let $x_{3}:=\min \left\{\alpha_{2}+6.1 / H, \alpha_{3}\right\}$. Using the Grönwall inequality (18), one obtains that for $x \in\left[\alpha_{2}, x_{3}\right]$

$$
g(x) \leq e^{\int_{\alpha_{2}}^{x}\left(s-\alpha_{2}\right)^{2} V_{2}(s)\left|V_{2}^{\prime}(s)\right|+\left(s-\alpha_{2}\right) V_{2}^{2}(s) d s} \int_{\alpha_{2}}^{x} \frac{1}{n+4} s U^{1-n}(s) d s
$$

Let $A:=\left\{x \in\left[\alpha_{2}, x_{3}\right] \mid U(s) \geq\left(H^{3} / 100\right)\left(s-\alpha_{2}\right)^{2}\right.$ on $\left.\left[\alpha_{2}, x\right]\right\}$. Note that $A$ in nonempty and closed. The estimate on $\left\|V-V_{2}\right\|$ implies that $V_{2}<1.1 H,\left|V_{2}^{\prime}\right|<\frac{H^{2}}{2}$, for $x \in A$ one obtains

$$
g(x) \leq e^{70}(C+12.2) 100^{n-1} H^{2-3 n} \frac{\left(x-\alpha_{2}\right)^{3-2 n}}{3-2 n} .
$$

Since $x-\alpha_{2}<6.1 / H$, for $H$ large enough and $x \in A$

$$
g(x) \leq \frac{\varepsilon}{400} H^{-3 / 2}
$$

Therefore $\left|U(x)-V_{2}(x)\right|<\frac{\varepsilon}{800} H^{-3 / 2}\left(x-\alpha_{2}\right)^{2}$ for $x \in A$ which implies that $U(x)>$ $V_{2}(x)-\frac{\varepsilon}{800} H^{-3 / 2}\left(x-\alpha_{2}\right)^{2}>\frac{H^{3}}{100}\left(x-\alpha_{2}\right)^{2}$. Therefore $A$ is open in $\left[\alpha_{2}, x_{3}\right]$. Since $A$ is also closed, $A=\left[\alpha_{2}, x_{3}\right]$. Arguing as at the end of the proof of Lemma 15 one obtains that $x_{3}=\alpha_{3}$ and $\alpha_{3}-\alpha_{2}>5 / H$ which concludes the proof.

5.2. Bounding $U-V$ for more than one lap. For an odd number $k$ we introduce:

$$
\gamma_{k}:=\inf \left\{\gamma \mid \tilde{\gamma} \in\left[\gamma,-H^{3} / 4+H\right] \Longrightarrow \tilde{\gamma} \in S_{k}^{+}(H, 0) \text { and } U\left(\alpha_{k+1}(H, \tilde{\gamma}, 0)\right)>0\right\}
$$

By repeating the argument needed to prove the previous lemmas one obtains:

Lemma 26. Let $1<n<3 / 2, \varepsilon \in(0,1)$, and $k$ be an odd number. Then there exists $H_{k}$ such that if $H \geq H_{k}$, 
a) $-H^{3} / 4+H \in S_{k}^{+}(H, 0)$ and hence $\gamma_{k}$ is well defined.

b) If $U(0)=V(0)=H, U^{\prime}(0)=V^{\prime}(0)=0, U^{\prime \prime}(0)=V^{\prime \prime}(0)=\gamma \in\left(\gamma_{k},-H^{3} / 4+\right.$ $H]$ and $U$ and $V$ are the resulting solutions of (12) and (6) respectively then $\|U-V\|_{\left[0, \alpha_{k}\right]}<\varepsilon H^{-7 / 2}$.

Furthermore, $5(k-1) / H<\alpha_{k}<6.1(k-1) / H$.

5.3. The Shooting Argument. We are now in a position to begin the shooting argument.

Lemma 27. Let $1<n<3 / 2$. Let $S_{3}^{+}(H, 0)$ be as defined in (29) and $\gamma_{3}$ be as defined in (31). Then for all $H$ large enough $\gamma_{3} \in S_{3}^{+}(H, 0)$.

Proof. Let $H$ be large enough so that the estimates of Lemma 26 hold with $\varepsilon=1 / 2$. Let $S_{3}^{+}=S_{3}^{+}(H, 0)$. Further, if $U$ is a solution of (12) with initial data $U(0)=H$, $U^{\prime}(0)=0$, and $U^{\prime \prime}(0)=\gamma$, we will denote the local extrema of $U$ by $0<\alpha_{2}(\gamma)<$ $\alpha_{3}(\gamma)<\ldots$

From the definition of $\gamma_{3},\left(\gamma_{3},-H^{3} / 4+H\right] \subset S_{3}^{+}$. In other words $\alpha_{4}(\gamma)$ exists for all $\gamma \in\left(\gamma_{3},-H^{3} / 4+H\right]$. We would like to conclude that by continuous dependence of solutions on initial data $\alpha_{4}\left(\gamma_{3}\right)$ also exists. The concern is that for $U$, the solution of the (12) with $U\left(x_{0}\right)=H, U^{\prime}\left(x_{0}\right)$ and $U^{\prime \prime}\left(x_{0}\right)=\gamma_{3}, U\left(\alpha_{2}\left(\gamma_{3}\right)\right)=0$ and then $\alpha_{4}\left(\gamma_{3}\right)$ does not exist. To exclude that possibility we provide for all $\gamma \in\left(\gamma_{3},-H^{3} / 4+H\right]$ a lower bound on $U\left(\alpha_{2}(\gamma)\right)$, where $U$ is the solution of (12) with $U\left(x_{0}\right)=H, U^{\prime}\left(x_{0}\right)=0$, and $U^{\prime \prime}\left(x_{0}\right)=\gamma$. Once

By Lemma 1 the local minima of $U$ are decreasing, and so there exists $\zeta \in\left(\alpha_{3}(\gamma)\right.$, $\left.\alpha_{4}(\gamma)\right)$ such that $U(\zeta)=U\left(\alpha_{2}(\gamma)\right)$. We now obtain a lower estimate on $U\left(\alpha_{2}(\gamma)\right)-$ $U\left(\alpha_{4}(\gamma)\right)$. By integrating (15) between $\alpha_{2}(\gamma)$ and $\zeta$ and using integration by parts one obtains

$$
\begin{aligned}
\frac{1}{2} U^{\prime}(\zeta)^{2} & =\int_{\alpha_{2}(\gamma)}^{\zeta}\left(\gamma+\frac{H^{3}}{3}-\frac{U^{3}}{3}-\frac{1}{n+4} \int_{0}^{x} s U(s)^{1-n} d s\right) U^{\prime}(x) d x \\
& =-\frac{U\left(\alpha_{2}(\gamma)\right)}{n+4} \int_{\alpha_{2}(\gamma)}^{\zeta} x U(x)^{1-n} d x+\int_{\alpha_{2}(\gamma)}^{\zeta} \frac{x U(s)^{2-n}}{n+4} d x \\
& =\frac{1}{n+4} \int_{\alpha_{2}(\gamma)}^{\zeta} x U(x)^{1-n}\left(U(x)-U\left(\alpha_{2}(\gamma)\right)\right) d x
\end{aligned}
$$

The estimates of Lemma 26, along with properties of approximating function $V$ imply that, $U>H / 2$ on a subinterval of $\left(\alpha_{2}(\gamma), \zeta\right)$ of length greater that $1 / H$ and that $U\left(\alpha_{2}(\gamma)\right)<1$. Therefore, for $H$ large,

$$
\frac{1}{2} U^{\prime}(\zeta)^{2}>\frac{1}{6} \frac{1}{H} \frac{H^{1-n}}{4}=\frac{H^{-n}}{24}
$$

Therefore $U^{\prime}(\zeta)<-H^{-n / 2} / 4$. From (13) follows that $U^{\prime \prime}<\frac{H^{3}}{10}$, and hence $U^{\prime}<$ $-H^{-n / 2} / 8$ on $\left(\zeta, \zeta+H^{-n / 2-3}\right)$. Therefore $\alpha_{4}\left(\gamma_{3}\right)>\zeta+H^{-n / 2-3}$ and

$$
U\left(\alpha_{4}(\gamma)\right)-U(\zeta)=\int_{\zeta}^{\alpha_{4}(\gamma)} U^{\prime}(x) d t<-H^{-n / 2-3} \frac{H^{-n / 2}}{8}=-\frac{H^{-n-3}}{8}
$$


Thus $U\left(\alpha_{2}(\gamma)\right)=U(\zeta)>H^{-3-n} / 8$ for all $\gamma \in\left(\gamma_{3},-H^{3} / 4+H\right]$. Continuous dependence on initial data (arguing as in the proof of Theorem 19) implies that for $\gamma=\gamma_{3}, U\left(\alpha_{2}\left(\gamma_{3}\right)\right)>0$ and that $\alpha_{4}\left(\gamma_{3}\right)$ exists. This proves $\gamma_{3} \in S_{3}^{+}$(and that $\left.\gamma_{3}>\gamma_{1}\right)$.

Corollary 28. Let $1<n<3 / 2$. For every odd $k>0$, for all H large enough $\gamma_{k} \in S_{k}^{+}$.

To prove this, it suffices to prove that $U\left(\alpha_{k-1}\left(\gamma_{k}\right)\right)>0$. Once this is known, $U\left(\alpha_{j}\left(\gamma_{k}\right)\right)>0$ for all even $j<k-1$ by Lemma 1 and so $\alpha_{k+1}\left(\gamma_{k}\right)$ exists as desired. The proof that $U\left(\alpha_{k-1}\left(\gamma_{k}\right)\right)>0$ is very similar to the proof of Lemma 27 and so we omit it.

Given any odd number $k$, we can now prove the existence of a zero-contact-angle selfsimilar solution, with $k$ local maxima, that is symmetric about $x=0$ and blows up at time $T=1$.

Theorem 29. Let $1<n<3 / 2$ and $k$ be an odd positive number. Then if $H$ is large enough there exists $\gamma$ such that the solution of (12) with $U(0)=H, U^{\prime}(0)=0$, and $U^{\prime \prime}(0)=\gamma$ has exactly $k$ local maxima, has support $[-L, L]$ and $U^{\prime}( \pm L)=0$.

Proof. Let $S_{k}^{+}=S_{k}^{+}(H, 0)$ and $S_{k}^{-}=S_{k}^{-}(H, 0)$ as in (29). It suffices to show that $\gamma_{k} \in S_{k}^{+} \cap S_{k}^{-}$. We have proven the claim for $k=1$ in Theorem 19; the proof for $k>1$ is quite similar in spirit.

Let $H$ be large enough so that the claims in Lemma 26 hold with $\varepsilon=1 / 2$. Corollary 28 ensures that $\gamma_{k} \in S_{k}^{+}$. The estimates of Lemma 26 and the properties of the approximating $V$ imply that $U^{\prime \prime}\left(\alpha_{k+1}\left(\gamma_{k}\right)\right)>1$. If $U\left(\alpha_{k+1}\left(\gamma_{k}\right)\right)$ were greater than 0 , then by continuous dependence on initial data there would exists a neighborhood $O$ of $\gamma_{k}$ such $O \subset S_{k}^{+}$and $U\left(\alpha_{k+1}(\gamma)\right)>0$ for all $\gamma \in O$, which would contradict the definition of $\gamma_{k}$. Therefore $\alpha_{k+1}\left(\gamma_{k}\right)=0$ and hence $\gamma_{k} \in S_{k}^{+} \cap S_{k}^{-}$which completes the proof.

To prove the analogous result for $k$ even we need to consider different kind of initial data. For $k$ odd the solution has a global maximum of size $H$ at $x=0$. As $x$ increases, $U$ then "oscillates" with subsequent local maxima having values close to $H$ and and subsequent local minima having values that get closer and closer to zero until $U$ touches down: on $[-L, L], U$ looks (approximately) like $k$ periods of a nonnegative periodic steady state.

For $k$ even, the solutions have a local minimum at $x=0$. To find multi-bump selfsimilar solutions that blow up at time $T=1$, we will initial data where $U(0)$ is small and $U^{\prime \prime}(0)$ is large. The solution then has a large global maximum and the solution then oscillates until it touches down.

Theorem 30. For any even positive $k$, and for all $\theta$ large enough there exists $h>0$ such that the solution of (12) with initial data $U(0)=h, U^{\prime}(0)=0$, and $U^{\prime \prime}(0)=\theta$, has exactly $k$ positive extrema and touches down with zero contact angle.

Proof. (Sketch) Given $\theta$ large, let $H=\sqrt[3]{12 \theta}$. Let $I=\left(0, H^{-3}\right)$. Note that solutions of the equation (6) with initial data $V(0)=h \in I, V^{\prime}(0)=0, V^{\prime \prime}(0)=\theta$, oscillate 
between $h$ and a value close to $H$. Applying the same techniques as before one can show that $U$ and $V$ stay close for a number of periods of $V$ (when $\theta$ is large enough). That enables one to argue via a shooting argument as before.

We believe that present techniques are sufficient to provide multibump analogue of Corollaries 14 and 22. Moreover, in [25, Figure 7] Witelski et al. presented computational evidence suggesting that as the number of bumps tends to infinity, $k \rightarrow \infty$, that the profile of the selfsimilar blow-up solution tends that of a configuration of $k$ zero-contact-angle droplet steady states with "touching" supports ${ }^{2}$.

\section{CONCLUSIONS AND FUTURE DIRECTIONS}

We studied selfsimilar blow-up solutions of the unstable thin-film equations with critical powers of nonlinearities $u_{t}=-\left(u^{n} u_{x x x}+u^{n+2} u_{x}\right)_{x}$. We have shown that for $n \geq 3 / 2$ there are no selfsimilar blow-up solutions with zero contact angles. For $n \in(0,3 / 2)$ the thin-film equation possesses a family of symmetric selfsimilar solutions with zero contact angles. The profiles, $U$, of these solutions can have one or more local maxima. Their global maximum one time unit before blow up must be greater then $\max \left\{1,(3-2 n)^{-2 / 11}\right\}$. We have also shown that for every $H>$ $\max \left\{40,3(3-2 n)^{-2 / 11}\right\}$ there exists a single-bump blowup profile, with maximum equal to $H$, that will blow up at $T=1$. What is the least value of $H, H_{\min }(n)$, for which such selfsimilar profiles exist is open. Witelski, Bertozzi and Bernoff [25] gave a formal argument in the case $n=1$ that if $U$ is the single-bump profile with $U(0)=H_{\min }(1)$ then $U^{\prime \prime}( \pm L)=0$ at the contact line $x= \pm L$. We believe that the same is true for all $0<n<3 / 2$.

An important open problem is the uniqueness (up to translation) of symmetric one-bump solutions given their maximal height. Lemma 5 provides a comparison result between a solution $U$ of (12) and a solution $V$ of (6). If there was an analogous comparison result between two solutions $U_{1}$ and $U_{2}$ of (12), uniqueness would follow. However, computations show that such a result is false in general. Nevertheless it may be possible to prove a more restrictive type of comparison result that would apply only to $\xi=0$ and $\gamma_{1,2}$ near $-H^{3} / 4$, which should be enough for uniqueness. Another open problem is to show that all selfsimilar zero-contact-angle solutions (with connected support) have to be symmetric.

The scale invariance of the equation is such that that the steady states centered at 0 with connected support and zero contact angles are dilation invariant and all have the same mass. We have shown that the profiles of selfsimilar blowup solutions are pointwise above the (centered) droplet steady states of the same height (except at the maximum where they are equal), and hence have greater mass. We have also remarked that analogous statement is true for selfsimilar spreading solutions whose profile (always single-bumped) is below the profile of the (centered) droplet steady state of equal height. Furthermore we know that for all $0<n<3$ initial data of mass less than $M_{c}$

\footnotetext{
${ }^{2}$ That is, if there are three droplets then the support of the configuration would be $[-L-L / 2, L+$ $L / 2]$ with the individual droplets supported on $[-L-L / 2,-L / 2],[-L / 2, L / 2]$, and $[L / 2, L+L / 2]$ respectively.
} 
yields a weak solution that exists for all time, and hence no blowup occurs. Numerical experiments of Witelski, Bernoff, and Bertozzi [25] suggest that for $n=1$ generic initial data of mass greater than $M_{c}$ yield solutions that blow up in finite time. We expect this is true for all $n>0$. Of course, not all initial data of mass greater than $M_{c}$ yield solutions that blow up. For example, if one takes two droplet steady states with disjoint support the resulting solution is time independent.

It would be very interesting to gain a more refined understanding of the dynamics of the blow up of these equations. For $n \in(0,3 / 2)$ it is expected that the blow up is governed by the selfsimilar blow-up solutions we have found. It is intuitively natural that multi-bump selfsimilar solutions should be unstable since a small perturbation could make one "bump" blow up sooner then the others. Witelski, Bernoff, and Bertozzi [25] show numerically that when $n=1$ the selfsimilar multi-bump blow-up solutions are linearly unstable. This fact has been rigorously established for the multi-bump selfsimilar blow-up solutions constructed here in [22].

On the other hand, single-bump solutions are expected to be stable (modulo invariances of the equation, that is translations and dilations) and hence govern the blow up of the equations. Again, linear stability analysis was demonstrated numerically for $n=1$ in [25], and is proven in [22] for the solutions constructed here.

Let us now assume that for each $H>0$ there exists at most one single-bump selfsimilar blow-up solution of maximum height $H$ that blows up at $T=1$. Denote $U_{H}$ this solution at time $t=0$. We have shown that $U_{H}$ is getting closer and closer to the zero-contact-angle droplet steady state, $V_{H}$, of the same maximal height and as $H \rightarrow \infty$ the mass of $U_{H}$ approaches $M_{c}$. It was shown numerically in [25] that for $n=1$ the mass of $U_{H}$ is decreasing as $H$ increases and the solution with smallest $H$ has the largest mass, that we denote by $M_{u}$. For $n=1, M_{u}$ is not much larger than $M_{c}$. It would be valuable to prove that the mass is indeed decreasing as $H$ increases and to provide a good upper bound on $M_{u}$. Our results suggest $M_{u} \rightarrow M_{c}$ as $n \rightarrow 3 / 2$.

Since selfsimilar behavior is expected to govern the blowup of these equations it is expected that even for initial data of large mass only a portion of mass somewhat greater than $M_{c}$ "participates" in the blow up. More precisely, we expect that at the blowup time mass concentration occurs in the following way: For a solution $u$ that blows up at time $T$ there exists a function $f \in L^{1}$ and points $x_{i} \in \mathbb{R}$ and real numbers $m_{i} \geq M_{c}, i=1, \ldots, N$ such that

$$
u(\cdot, t) \rightarrow f+\sum_{i=1}^{N} m_{i} \delta_{x_{i}} . \quad \text { as } \quad t \uparrow T
$$

Generically $N=1$ and $m_{1} \leq M_{u}$. If this picture is correct one could continue solutions past the blowup time by discarding the mass that has blown-up, not unlike the drops that have fallen off the ceiling. That is, by considering the initial problem with $f$ as initial data at time $T$.

Finally, studying the blowup of solutions for $n \geq 3 / 2$, when no zero-contact-angle selfsimilar blow up solutions exist, is another challenging problem. We believe that generic initial data with mass greater than $M_{c}$ yield solutions that blow up in finite 
time in a focusing selfsimilar manner. However, since there are no zero-contact-angle selfsimilar solutions any blow up solution must involve an inner (selfsimilar) solution and an outer solution to which the selfsimilar solution matches. It is not clear to us how the solution of the initial value problem would select how much mass will be carried by the selfsimilar portion and how much mass would remain behind in the outer solution. Indeed, this is also an open problem for the $0<n<3 / 2$ initial value problem.

Acknowledgments. This work was partially supported by NSERC grant number 250305-02 and by MCP's Alfred P. Sloan fellowship. The authors thank Richard S. Laugesen for sharing his insights. The authors thank an anonymous referee for his careful reading and valuable comments.

\section{REFERENCES}

[1] E. Beretta. Selfsimilar source solutions of a fourth order degenerate parabolic equation. Nonlinear Anal.-Theor, 29(7):741-760, 1997.

[2] E. Beretta, M. Bertsch, and R. Dal Passo. Nonnegative solutions of a fourth order nonlinear degenerate parabolic equation. Arch. Ration. Mech. Anal., 129:175-200, 1995.

[3] F. Bernis Finite speed of propagation and continuity of the interface for thin viscous flows. Adv. Differential Equations , 1(3):337-368, 1996.

[4] F. Bernis and R. Ferreira. Source-type solutions to thin-film equations: the critical case. Appl. Math. Lett., 12(7):45-50, 1999.

[5] F. Bernis, J. Hulshof, and J.R. King. Dipoles and similarity solutions of the thin-film equation in the half-line. Nonlinearity, 13(2):413-439, 2000.

[6] F. Bernis, L. A. Peletier, and S. M. Williams. Source type solutions of a fourth order nonlinear degenerate parabolic equation. Nonlinear. Anal.-Theor., 18(3):217-234, 1992.

[7] A.J. Bernoff and T.P. Witelski. Linear stability of source-type similarity solutions of the thin film equation. Appl. Math. Lett., 15(5)599-606, 2002.

[8] A. L. Bertozzi and M. C. Pugh. Long-wave instabilities and saturation in thin-film equations. Comm. Pure Appl. Math., 51:625-661, 1998.

[9] A. L. Bertozzi and M. C. Pugh. Finite-time blow-up of solutions of some long-wave unstable thin film equations. Indiana Univ. Math. J., 49(4):1323-1366, 2000.

[10] M. Bowen, J. Hulshof, and J.R. King. Anomalous exponents and dipole solutions for the thin-film equation. SIAM J. Appl. Math., 62(1):149-179 (electronic), 2001.

[11] M. Bowen and J.R. King. Asymptotic behaviour of the thin film equation in bounded domains. European J. Appl. Math., 12(2):135-157, 2001.

[12] R. Dal Passo, L. Giacomelli, and A. Shishkov. The thin film equation with nonlinear diffusion. Comm. Partial Differential Equations 26(9-10):1509-1557, 2001.

[13] P. Ehrhard. The spreading of hanging drops. J. Colloid. Interf. Sci., 168:242-246, 1994.

[14] R. Ferreira and F. Bernis. Source-type solutions to thin-film equations in higher dimensions. European J. Appl. Math., 8:507-524, 1997.

[15] G. Grün, Droplet spreading under weak slippage: existence for the Cauchy problem. to appear in Comm.Part.Diff.Equations, 2004.

[16] J. Hulshof and A.E. Shishkov. The thin film equation with $2 \leq n<3$ : finite speed of propagation in terms of the $L^{1}$-norm. Adv. Differential Equations , 3(5):625-642, 1998.

[17] R. S. Laugesen and M. C. Pugh. Properties of steady states for thin-film equations. European. J. Appl. Math., 11(3):293-351, 2000.

[18] D. S. Mitrinović, J. E. Pečarić, and A. M. Fink. Inequalities involving functions and their integrals and derivatives, volume 53 of Mathematics and its Applications (East European Series). Kluwer Academic Publishers Group, Dordrecht, 1991.

[19] T. G. Myers. Thin films with high surface tension. SIAM Rev., 40(3):441-462 (electronic), 1998. 
[20] A. Oron, S. H. Davis, and S. G. Bankoff. Long-scale evolution of thin liquid films. Rev. Mod. Phys., 69(3):931-980, Dec 1997.

[21] F. Otto, Lubrication approximation with prescribed nonzero contact angle. Comm. Partial Differential Equations 23(11-12):2077-2164, 1998.

[22] D. Slepčev. Linear stability of selfsimilar solutions of long-wave unstable thin-film equations. in preparation.

[23] Béla v. Sz. Nagy. Über Integralungleichungen zwischen einer Funktion und ihrer Ableitung. Acta Univ. Szeged. Sect. Sci. Math., 10:64-74, 1941.

[24] J. B. van den Berg, M. Bowen, J. R. King, and M. M. A. El-Sheikh. The self-similar solution for draining in the thin film equation. European J. Appl. Math 15(3):329-346, 2004.

[25] T. P. Witelski, A. J. Bernoff, and A. L. Bertozzi. Blowup and dissipation in a critical-case unstable thin film equation. European J. Appl. Math. 15(2):223-256, 2004.

[26] T. P. Witelski and A. J. Bernoff. Stability of self-similar solutions for van der Waals driven thin film rupture. Phys. Fluids 11(9):2443-2445, 1999.

[27] T. P. Witelski and A. J. Bernoff. Dynamics of three-dimensional thin film rupture. Phys. D 147(1-2):155$176,2000$.

[28] Zhang, W. W. and Lister, J. R. Similarity solutions for van der Waals rupture of a thin film on a solid substrate. Phys. Fluids 11(9):2454-2462, 1999.

Department of Mathematics, University of ToRonto, ToRonto, ON M5S 3G3

Department of Mathematics, University of ToRonto, ToRonto, ON M5S 3G3 